\title{
From Hierarchies to Levels: New Solutions for Games
}

\section{with Hierarchical Structure}

\author{
Álvarez-Mozos, M. ${ }^{1}$, van den Brink, R. ${ }^{2}$, van der Laan, G. ${ }^{3}$, and Tejada, O. ${ }^{* 4}$ \\ ${ }^{1}$ Departament de Matemàtica Econòmica, Financera i Actuarial, Universitat de Barcelona, Spain. \\ ${ }^{2,3}$ Department of Econometrics and Tinbergen Institute, VU Amsterdam, The Netherlands. \\ ${ }^{4}$ CER-ETH Center of Economic Research, ETH Zurich, Switzerland.
}

Published in International Journal of Game Theory (2017)

Published version available at http://link.springer.com

DOI: $10.1007 / \mathrm{s} 00182-017-0572-\mathrm{z}$

\begin{abstract}
Recently, applications of cooperative game theory to economic allocation problems have gained popularity. In many of these problems, players are organized according to either a hierarchical structure or a levels structure that restrict the players' possibilities to cooperate. In this paper, we propose three new solutions for games with hierarchical structure and characterize them by properties that relate a player's payoff to the payoffs of other players located in specific positions in the hierarchical structure relative to that player. To define each solution, we consider a certain mapping that transforms the hierarchical structure into a levels structure, and then we apply the standard generalization of the Shapley Value to the class of games with levels structure. Such transformation mappings are studied by means of properties that relate a player's position in both types of structure.
\end{abstract}

Keywords: TU-game; hierarchical structure; levels structure; Shapley Value; axiomatization

JEL classification: C7, C70.

${ }^{*}$ Corresponding author. E-mail: toriol@ethz.ch. 


\section{Introduction}

Many economic allocation problems are modeled by a cooperative game. A cooperative game with transferable utility, or simply a game, is a pair consisting of a finite set of players and a mapping that associates a real number with each subset (or coalition) of players. Each of these numbers represents the aggregate benefit that the players can obtain by cooperating within the corresponding coalition. In many of these problems, there is a natural structure according to which the players are arranged, which also needs to be taken into account. For instance, in water allocation problems (Ambec and Sprumont, 2002) or in polluted river cost allocation problems (Ni and Wang, 2007), a graph is used to describe the agents' location along the river. Problems with an implicit order of the players are discussed e.g. in Littlechild and Owen (1973), Curiel et al. (1989), Graham et al. (1990), and Maniquet (2003).

In this paper, we deal with games with hierarchical structure. They consist of a game plus a directed graph in the form of a (directed) tree that describes the organizational design of the set of players. Most political, economic or military organizations are organized in a hierarchical structure, which makes the study of such structures a relevant topic in economic theory, both from a normative and a positive viewpoint. The study of the properties that certain solutions for games with hierarchical structure satisfy has received particular attention in the literature, and many important contributions to this model - as well as to more general, closely related models - have been made. The main goal of this paper is to make further progress in this direction.

The class of games with permission structure consists of all pairs made up of a game and a directed graph (or digraph). Hence, it contains the class of games with hierarchical structure. For games with permission structure, Gilles et al. (1992) assume that any player needs permission from all her predecessors in the digraph in order to cooperate with other players. Therefore, a coalition is feasible, i.e. it can cooperate, if and only if for every player in the coalition, all her predecessors also belong to the coalition (see also van den Brink and Gilles, 1996). The permission restricted game assigns to each coalition the worth of its largest feasible subset in the

original game. The Permission Value is then the solution on the class of games with permission structure that assigns to each element in the class the Shapley Value of its permission restricted 
game. ${ }^{1}$ On the class of games with hierarchical structure, the so-called Average Tree Permission Value (van den Brink et al., 2015) is obtained by taking the average of all hierarchical outcomes (see Demange, 2004) of the permission restricted game on the underlying undirected graph. For games with an acyclic digraph, the Precedence Shapley Value (Faigle and Kern, 1992) is obtained by taking the average of all marginal vectors corresponding to the permutations that satisfy the property that every player comes before any of her predecessors in the digraph.

Another class of games with restricted cooperation that has long been studied is that of games with coalition structure, which was introduced by Aumann and Drèze (1974) and has been studied later on by Owen (1977) and Hart and Kurz (1983), among others. In that model, the game is enriched with a partition of the player set that aims at describing situations where some players are more closely related to each other than to other players. The class of games with levels structure introduced by Winter (1989) constitutes a natural generalization of the class of games with coalition structure. In a game with levels structure, there is an ordered set of partitions, with the first level being a non-trivial partition of the player set, each level being coarser than the next level, and the last level being the partition of all singletons. Hence, the latter games describe situations where within any coalition at any level, some players are closer to each other than to other players.

The main contribution of the present paper is to propose and characterize three new solutions for games with hierarchical structure. Each of the suggested solutions is based on a two-stage procedure of the following type: first, the hierarchical structure is converted into a levels structure; second, the Shapley Levels Value (Winter, 1989) is applied to the game with the levels structure obtained in the first stage. To the best of our knowledge, conversion mappings of this type have not been sufficiently studied despite the fact that they may be useful for the comparison of institutions with different organizational layouts. Our paper adds to the comprehension of this matter and connects the literature on games with hierarchical structure to the literature on games with levels structure.

The set of mappings that convert trees into levels structures is very large. Here, we only consider conversion mappings based on a procedure that starts by setting the grand coalition

\footnotetext{
${ }^{1}$ This is called the conjunctive approach. In contrast, in the disjunctive approach of Gilles and Owen (1994), every player only needs permission from one of her predecessors in the digraph (see also van den Brink, 1997). For games with hierarchical structure, the two approaches coincide.
} 
as the least refined partition and continues with the iterative application of the following two steps. ${ }^{2}$ First, each element of the previous partition is divided into two subsets, namely the root of the subtree on that element of the partition and the set of all other players therein. Second, each of the subsets is further divided in several sets, so that each consists of a player plus her subordinates. ${ }^{3}$ The procedure just described underlying the conversion mappings we use to construct our three solutions has value in its own right. Nevertheless, to further justify such a procedure, we shall resolve it by a collection of properties that any partition of the player set might satisfy in accordance with a given tree.

Both mathematical objects, directed trees and levels structures, are useful each to capture a particular type of restriction to the players' cooperation possibilities that may exist in an organization. On the one hand, a directed tree expresses a hierarchical configuration. In a firm, for instance, an employee might not be allowed to take some decision without the approval of her superior-being her predecessor in the tree-, as is assumed when applying the Permission Value. According to Demange (2004), a hierarchical structure is the organizational form that maximizes stability. With any player in a hierarchical structure, we can associate a so-called team of players, which consists of the player herself and the players who are below her in the hierarchy. Demange (2004) also argues that in a hierarchical structure, teams are the relevant units in the decision-making process. A levels structure, on the other hand, organizes a set of players into a series of nested partitions. For instance, a firm might be structured in different divisions, which, in turn, might be divided in different departments, in which employees work. To take a decision, it might be that, first, all workers of each department have to reach a consensus, second, all departments of each division have to reach a consensus, and, third and last, all divisions have to reach an agreement, so that the firm as a whole takes the ultimate decision.

Each of the three solutions for games with hierarchical structure that we introduce builds on a particular conversion mapping. For the first solution, the entire levels structure resulting from

\footnotetext{
${ }^{2}$ Formally, we do not consider the grand coalition as an element of a levels structure. Considering it here, however, facilitates the exposition of the procedure upon which our conversion mappings are based.

${ }^{3} \mathrm{By}$ construction, this procedure may entail some loss of information when some leaf of the tree has no siblings, i.e., there are no other players having the same predecessor in the hierarchy. However, on the set of trees where each leaf has at least one sibling, the procedure establishes a bijection between the latter set and the set of levels structures.
} 
the procedure described above is used. For the other two solutions, by contrast, strict subsets of the collection of all levels are used. The second solution uses the so-called team levels, composed of teams and singletons. The third solution uses the so-called sibling levels, that arise when siblings at a given height of the hierarchy become singletons. The characterization results we provide for all three solutions spell out their differences from an axiomatic viewpoint. Such a comparative analysis is extended in Section 6 to the solutions from the literature on games with hierarchical structure mentioned before. Our axiomatizations revolve around the role of players who are either very weak or very strong in the game, in a sense that we will explain shortly.

To elaborate, it is instructive to recall some properties of the Permission Value (PV) and the Precedence Shapley Value (PSV), theirs being two of the most established solutions for games with hierarchical structure. On the one hand, the PSV - but not the PV-satisfies the Null Player Property, which requires that a null player has to earn a zero payoff irrespective of her position in the tree. A null player does not contribute to creating any worth in the game, and thus is a "weak" player. On the other hand, the PV-but not the PSV-satisfies the Necessary Player Property, which requires that in a monotone game, a necessary player has to earn at least as much as anybody else. A player is necessary in the game if the worth of any coalition not containing her is zero, and thus is a "strong" player.

Together with Efficiency and Additivity, the Null Player Property and the Necessary Player Property single out the Shapley Value of the game. This implies that the hierarchical structure will be ignored if we simultaneously require the four properties. Throughout the paper, we impose Efficiency and Additivity, as both properties are standard in the literature. Moreover, we take the normative stance that except for null players, payoffs should depend on both the marginal contributions of the players - i.e., the game - and the position of the players in the tree. This constrains us to impose the Null Player Property and waive the Necessary Player Property. Instead of the latter, we consider several weaker axioms, all of which set as an upper bound to a player's payoff the total payoff of a certain related coalition (her siblings, her team, her successors' team, or her siblings' successors) containing a necessary player. ${ }^{4}$ For each of these upper bound properties, we then consider the corresponding lower bound property, which results

\footnotetext{
${ }^{4}$ We will show that there is a sense in which the bound properties that we consider do indeed balance out a player's position in the hierarchy with her productivity in a reasonable way.
} 
from reversing the inequality and the identity of the necessary player. It turns out that with the help of some auxiliary properties, we are able to single out each of the three solutions for games with hierarchical structure that we propose by choosing different combinations of lower and upper bound properties. ${ }^{5}$

The paper is organized as follows: Section 2 is a preliminary section on games, directed graphs, and levels structures. In Section 3 we introduce and characterize the procedure described above, which assigns a levels structure to every hierarchical structure represented by a directed tree. In Section 4 we introduce and axiomatize our first solution for games with hierarchical structure. This solution uses all the information contained in the levels structure obtained from the conversion mapping of Section 3. In Section 5 we define and axiomatize two alternative solutions for these games. These solutions use proper subsets of the levels structure. In Section 6 we first review and assess the properties introduced throughout the paper. Then we compare the three new solutions with some existing solutions from the literature. Section 7 contains the concluding remarks. All proofs can be found in the online Appendix - see Supplementary Material.

\section{Preliminaries}

\section{$2.1 \quad$ Games}

Let a finite non-empty set $\Omega \subset \mathbb{N}$ of potential players be given. Then, a cooperative game with transferable utility, or simply a game, is a pair $(N, v)$, where $\emptyset \neq N \subseteq \Omega$ is a finite set of players and $v: 2^{N}=\{S: S \subseteq N\} \rightarrow \mathbb{R}$ is a characteristic function, with $v(\emptyset)=0$. For any coalition $S \subseteq N, v(S)$ represents the worth of coalition $S$, i.e., the total payoff that members of the coalition can obtain by agreeing to cooperate. We denote the collection of all games by $\mathcal{G}$. For the sake of readability, we henceforth slightly abuse notation and write $T \cup i$ and $T \backslash i$ instead of $T \cup\{i\}$ and $T \backslash\{i\}$ for $T \subseteq N$ and $i \in N$, respectively. We use the $|\cdot|$ operator to denote the cardinality of a finite set.

Given $(N, v) \in \mathcal{G}$ and $i \in N$, we say that $i$ is a null player in $(N, v)$ if $v(T \cup i)-v(T)=0$

\footnotetext{
${ }^{5}$ Among the auxiliary properties, the one that requires that teams have to be autonomous in distributing their payoffs stands out. Such a property is used in the axiomatization of the first two solutions.
} 
for all $T \subseteq N \backslash i$, and we say that $i$ is a necessary player in $(N, v)$ if $v(T)=0$ for all $T \subseteq N \backslash i$. A game $(N, v) \in \mathcal{G}$ is monotone if $v(T) \leq v(R)$ for all $T \subseteq R \subseteq N$. We denote by $\mathcal{G}_{M} \subset \mathcal{G}$ the class of all monotone games. For each nonempty $T \subseteq N$, the unanimity game $\left(N, u_{T}\right)$ is given by $u_{T}(R)=1$ if $T \subseteq R$, and $u_{T}(R)=0$ otherwise. It is well-known that every game $(N, v) \in \mathcal{G}$ can be written in a unique way as a linear combination of unanimity games $\left(N, u_{T}\right)$ by $v=\sum_{\emptyset \neq T \subseteq N} \Delta_{T}(v) u_{T}$, where $\Delta_{T}(v)=\sum_{R \subseteq T}(-1)^{|T|-|R|} v(R)$ are the Harsanyi dividends (Harsanyi, 1959).

A solution on $\mathcal{G}$ is a map, $f$, that assigns a unique vector $f(N, v) \in \mathbb{R}^{N}$ to every $(N, v) \in \mathcal{G}$, where $f_{i}(N, v)$ represents the payoff to player $i \in N$. A permutation of $N$ is a bijective map $\pi: N \rightarrow N$. Let $\Pi(N)$ denote the set of permutations of $N$. Given $\pi \in \Pi(N)$ and $i \in N$, let $\pi^{-1}[i]$ indicate the set of players ordered before $i$ in permutation $\pi$, i.e., $\pi^{-1}[i]=\{j \in N: \pi(j)<\pi(i)\}$. The best-known solution on $\mathcal{G}$ is the Shapley Value (Shapley, 1953), which is defined for every $(N, v) \in \mathcal{G}$ and $i \in N$ by

$$
S h_{i}(N, v)=\frac{1}{|\Pi(N)|} \sum_{\pi \in \Pi(N)}\left(v\left(\pi^{-1}[i] \cup\{i\}\right)-v\left(\pi^{-1}[i]\right)\right) .
$$

\subsection{Games with hierarchical structure}

Given the set $\Omega$ of potential players, a directed graph (or digraph) is a pair $(N, D)$, where $\emptyset \neq N \subseteq \Omega$ is a finite set of nodes (representing the players) and $D \subseteq N \times N$ is a binary relation on $N$ (representing the hierarchy). Given $(N, D)$ and $T \subseteq N$, the digraph $\left(T, D_{T}\right)$ is the induced subgraph on $T$ given by $D_{T}=\{(i, j) \in D: i, j \in T\}$. For a given digraph $(N, D)$ and $i, j \in N$, a (directed) path from $i$ to $j$ is a sequence of distinct nodes $\left(i_{1}, \ldots, i_{m}\right)$ such that $i_{1}=i, i_{m}=j$, and $\left(i_{k}, i_{k+1}\right) \in D$ for $k=1, \ldots, m-1$. A digraph $(N, D)$ is a (rooted) directed tree with root $i_{0} \in N$ if there does not exist a player $j \in N$ with $\left(j, i_{0}\right) \in D$ and if there is exactly one directed path from the top-node $i_{0}$ to every other node. Note that, in particular, $(i, i) \notin D$ for all $i \in N$ if $(N, D)$ is a directed tree. We denote the set of all directed trees by $\mathcal{D}$.

For every $(N, D) \in \mathcal{D}$ and $i \in N$, the nodes in $S_{D}(i)=\{j \in N:(i, j) \in D\}$ are called the successors of $i$, and the nodes in $P_{D}(i)=\{j \in N:(j, i) \in D\}$ are called the predecessors of $i$. For a directed tree $(N, D) \in \mathcal{D}$ with root $i_{0}$, it holds that $P_{D}\left(i_{0}\right)=\emptyset$ and $\left|P_{D}(j)\right|=1$ for every $j \in N \backslash\left\{i_{0}\right\}$ and, accordingly, we denote by $p_{D}(j)$ the unique predecessor of $j \neq i_{0}$. Let $(N, \widehat{D})$ be the transitive closure of a digraph $(N, D)$, i.e., $(i, j) \in \widehat{D}$ if and only if there is a directed 
path from $i$ to $j$. The players in $\widehat{S}_{D}(i)=S_{\widehat{D}}(i)$ are called the subordinates of $i$, and the players in $\widehat{P}_{D}(i)=P_{\widehat{D}}(i)$ are called the superiors of $i$.

For a directed tree, we follow Demange (2004) and call the set $\widehat{S}_{D}(i) \cup i$ the team of player $i$, i.e. the team of $i$ consists of $i$ herself plus all her subordinates. We stress that the set $\widehat{P}_{D}(i)$, with $i \neq i_{0}$, is the set of nodes on the unique path from $i_{0}$ to $i$, excluding $i$ herself. The rank of $i$ in the hierarchy is the length of this path and is denoted by $l^{D}(i)$, i.e., for every $i \in N, l^{D}(i)=\left|\widehat{P}_{D}(i)\right|$. Whenever there is no possible confusion regarding $(N, D)$, we write $l(i)$ instead of $l^{D}(i)$. For every $(N, D) \in \mathcal{D}$, the depth of the hierarchy is given by $l(D)=\max _{i \in N} l(i)$. Further, given $(N, D) \in \mathcal{D}$ and $i \in N \backslash i_{0}$, the set of siblings of $i$ is denoted by $A(i)$ and is the set of players with the same predecessor as $i$, including $i$ herself, i.e., $A(i)=\left\{j \in N:\left(p_{D}(i), j\right) \in D\right\}$. Finally, for $T \subseteq N$ we denote $S_{D}(T)=\cup_{i \in T} S_{D}(i)$.

A game with hierarchical structure is a triple $(N, v, D)$, where $(N, v) \in \mathcal{G}$ and $(N, D) \in \mathcal{D}$. We denote by $\mathcal{G} \mathcal{D}$ the set of all games with hierarchical structure. A solution on $\mathcal{G} \mathcal{D}$ is a map, $f$, that assigns a vector $f(N, v, D) \in \mathbb{R}^{N}$ to every triple $(N, v, D) \in \mathcal{G D}$.

\subsection{Games with levels structure}

A partition of a finite set $N$ is a collection of subsets, $P \subseteq 2^{N}$, such that (i) $T \neq \emptyset$ for every $T \in P$, (ii) $\cup_{T \in P} T=N$, and (iii) for every $T, R \in P$ with $T \neq R, T \cap R=\emptyset$. Given $\Omega$, a levels structure is a pair $(N, \underline{B})$, where $\emptyset \neq N \subseteq \Omega,|N| \geq 2$, and, for some integer $m \geq 0$, $\underline{B}=\left(B_{1}, \ldots, B_{m+1}\right)$ is a sequence of $m+1$ partitions of $N$ such that (i) $B_{1}$ is a proper partition, i.e. $\left|B_{1}\right| \geq 2$, (ii) for each $r \in\{1, \ldots, m\}, B_{r}$ is coarser than $B_{r+1}$, i.e., for each $T \in B_{r}$ there is $U \subseteq B_{r+1}$ such that $T=\cup_{R \in U} R$, and (iii) $B_{m+1}=\{\{i\}: i \in N\}{ }^{6}$ The partition $B_{m+1}$ consisting of all singleton coalitions is added for notational convenience. For each $r \in\{1, \ldots, m+1\}$, the partition $B_{r}$ is called the $r$-th level of $\underline{B}$ and each $T \in B_{r}$ is called a union (of the $r$-th level). We denote by $\left(N, \underline{B}_{0}\right)$ the trivial levels structure, where $\underline{B}_{0}=(\{\{i\}: i \in N\})$ is the unique levels structure with $m=0$. The collection of all levels structures $(N, \underline{B})$, with $\emptyset \neq N \subseteq \Omega$, is denoted by $\mathcal{L}$.

Example 2.1. Let $N=\{1,2,3,4,5\}$ and $\underline{B}=\left(B_{1}, B_{2}, B_{3}\right)$ be given by $B_{1}=\{\{1,2\},\{3,4,5\}\}$, $B_{2}=\{\{1,2\},\{3\},\{4,5\}\}$, and $B_{3}=\{\{1\},\{2\},\{3\},\{4\},\{5\}\}$. Then, the pair $(N, \underline{B})$ is a levels

\footnotetext{
${ }^{6}$ Note that we do not exclude the possibility that $B_{r}=B_{r+1}$ for some $r \in\{1, \ldots, m\}$.
} 
structure, i.e., $(N, \underline{B}) \in \mathcal{L}$.

A game with levels structure is a triple $(N, v, \underline{B})$, where $(N, v) \in \mathcal{G}$ and $(N, \underline{B}) \in \mathcal{L}$. We denote by $\mathcal{G} \mathcal{L}$ the set of all games with levels structure. A solution on $\mathcal{G L}$ is a map, $f$, that assigns to every $(N, v, \underline{B}) \in \mathcal{G L}$ a vector $f(N, v, \underline{B}) \in \mathbb{R}^{N}$. The best-known solution on $\mathcal{G L}$ is the Shapley Levels Value, introduced by Winter (1989). ${ }^{7}$ This solution is based on the assumption that the levels structure restricts the admissible permutations. For a levels structure $(N, \underline{B}) \in \mathcal{L}$ with $\underline{B}=\left(B_{1}, \ldots, B_{m+1}\right)$, define the sets of permutations $\Omega_{r}(\underline{B})$, with $r \in\{0, \ldots, m\}$, starting with $\Omega_{0}(\underline{B})=\Pi(N)$, and then recursively for $r=1, \ldots, m$,

$$
\Omega_{r}(\underline{B})=\left\{\pi \in \Omega_{r-1}(\underline{B}): \forall T \in B_{r}, \forall i, j \in T \text { and } k \in N \text {, if } \pi(i)<\pi(k)<\pi(j) \text { then } k \in T\right\} .
$$

Therefore, $\Omega_{r}(\underline{B})$, with $r>0$, is the subset of permutations of $\Omega_{r-1}(\underline{B})$ such that the elements of each union of $B_{r}$ are consecutive. We let $\Omega(\underline{B})=\Omega_{m}(\underline{B})$ be the set of permutations that keep the agents of every union of every level consecutive. Then, the Shapley Levels Value $S h^{L}$ is the solution on $\mathcal{G L}$ defined for every $(N, v, \underline{B}) \in \mathcal{G} \mathcal{L}$ and $i \in N$ by

$$
S h_{i}^{L}(N, v, \underline{B})=\frac{1}{|\Omega(\underline{B})|} \sum_{\pi \in \Omega(\underline{B})}\left(v\left(\pi^{-1}[i] \cup i\right)-v\left(\pi^{-1}[i]\right)\right) .
$$

Note that the trivial levels structure $\left(N, \underline{B}_{0}\right)$ does not put any restriction on the order of players. Therefore, for every $(N, v) \in \mathcal{G}, S h^{L}\left(N, v, \underline{B}_{0}\right)=S h(N, v)$. A game with levels structure where $r=1$ corresponds to a game with coalition structure, as introduced in Aumann and Drèze (1974). It is easy to verify that the Shapley Levels Value yields the same payoff vector as the Owen Value (Owen, 1977) for the latter class of games.

\section{From Directed Trees to Levels Structures}

Next, we turn to the first relevant question of this paper: Is there a reasonable way to convert a given hierarchical structure into a levels structure? To answer it, we abstract for the moment from the analysis of how payoffs should be allocated in a game with hierarchical structure, and we focus on the relation between directed trees and levels structures. First, we propose a procedure to convert a directed tree into a collection of nested partitions. Second, we show that a mapping

\footnotetext{
${ }^{7}$ Other solutions for games with levels structure can be found in Álvarez-Mozos and Tejada (2011).
} 
that converts any directed tree into a levels structure satisfies some required properties if and only if any directed tree is mapped into a levels structure that is a subset of the collection of nested partitions obtained from our procedure.

Accordingly, we start by proposing a way to map any directed tree into a levels structure. Let $\mathcal{H}: \mathcal{D} \rightarrow \mathcal{L}$ be such that for every $(N, D) \in \mathcal{D}, \mathcal{H}(N, D)=\left(N, B^{D}\right)$ with

$$
B^{D}=\left(B_{1,1}^{D}, B_{1,2}^{D}, B_{2,1}^{D}, B_{2,2}^{D}, \ldots, B_{l(D), 1}^{D}, B_{l(D), 2}^{D}\right)
$$

defined for $r \in\{1, \ldots, l(D)\}$ by

$$
\begin{aligned}
& B_{r, 1}^{D}=\{\{i\}: l(i)<r\} \cup\left\{\widehat{S}_{D}(j): l(j)=r-1\right\} \quad \text { and } \\
& B_{r, 2}^{D}=\{\{i\}: l(i)<r\} \cup\left\{\widehat{S}_{D}(j) \cup j: l(j)=r\right\} .
\end{aligned}
$$

By definition, we obtain that $(i) B_{1,1}^{D}$ is a proper partition of $N$, (ii) $B_{1,2}^{D}$ is a refinement of $B_{1,1}^{D}$, and (iii) for every $r \in\{2, \ldots, l(D)\}$, the partition $B_{r, 1}^{D}$ is a refinement of $B_{r-1,2}^{D}$ and the partition $B_{r, 2}^{D}$ is a refinement of $B_{r, 1}^{D}$. Further, we note that $B_{l(D), 2}^{D}=\{\{i\}: i \in N\}$, since $\widehat{S}_{D}(j)=\emptyset$ for every $j \in N$ with $l(j)=l(D){ }^{8}$ As a consequence, $\left(N, B^{D}\right) \in \mathcal{L}$.

We illustrate the above definitions by an example that will be used throughout the paper.

Example 3.1. Consider the directed tree $(N, D) \in \mathcal{D}$, with $N=\{1, \ldots, 8\}$ and $D=\{(1,2),(1,3)$, $(2,4),(2,5),(3,6),(5,7),(5,8)\}$ - see the graph below. Then, $\left(N, B^{D}\right) \in \mathcal{L}$ is given by $B^{D}=$ $\left(B_{1,1}^{D}, \ldots, B_{3,2}^{D}\right)$, where

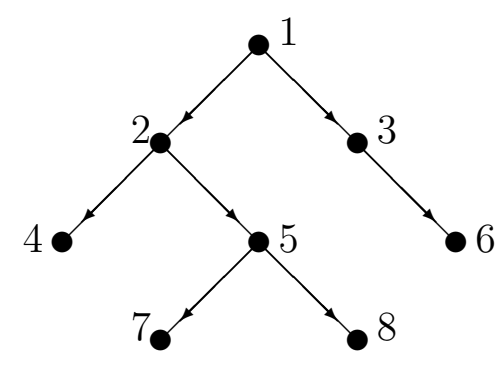

$$
\begin{aligned}
& B_{1,1}^{D}=\{\{1\},\{2,3,4,5,6,7,8\}\}, \\
& B_{1,2}^{D}=\{\{1\},\{2,4,5,7,8\},\{3,6\}\}, \\
& B_{2,1}^{D}=\{\{1\},\{2\},\{3\},\{4,5,7,8\},\{6\}\}, \\
& B_{2,2}^{D}=\{\{1\},\{2\},\{3\},\{4\},\{5,7,8\},\{6\}\}, \\
& B_{3,1}^{D}=\{\{1\},\{2\},\{3\},\{4\},\{5\},\{6\},\{7,8\}\}, \\
& B_{3,2}^{D}=\{\{1\},\{2\},\{3\},\{4\},\{5\},\{6\},\{7\},\{8\}\} .
\end{aligned}
$$

The object of the remaining part of this section is to provide a justification for the mapping $\mathcal{H}$ defined in Eqs. (3.2)-(3.4). First, note that the conversion mapping $\mathcal{H}$ respects the important

\footnotetext{
${ }^{8}$ Note that two consecutive partitions of $B^{D}$ might coincide.
} 
role of the teams, as highlighted by Demange (2004), in the following sense: At each even level $B_{r, 2}^{D}$, every player $i$ with $l(i)<r$ is a singleton in the partition, while each player with rank $l(i)=r$ in the hierarchy forms a union with all other players in his team. Furthermore, at each odd level $B_{r, 1}^{D}$, every player $i$ with $l(i)<r$ is a singleton in the partition, while the team of each player with rank $l(i)=r$ in the hierarchy forms a union with the teams of all her siblings. Accordingly, we call every even level a team level and every odd level a sibling level.

Next, we show that the mapping $\mathcal{H}$ can essentially be characterized by five properties. These properties connect the agents' position in a directed tree to their participation in a given union of a partition of the player set. Although we only consider properties that apply to a single partition of the player set, such properties can straightforwardly be generalized to a levels structure.

The properties considered follow two principles. For a given player in the directed tree, the first principle considers her most natural companions in the partition. In order of preference, these are: (i) her team, (ii) her siblings, and (iii) all other players. The second principle takes a certain equality notion into account, by requiring that under some additional conditions, two agents with the same rank in the directed tree are treated equally in the partition. Given a partition $P$ of $N$ and $i \in N$, let $P_{i}$ denote the element of $P$ to which player $i$ belongs.

Definition 3.1. Given a directed tree $(N, D) \in \mathcal{D}$, a partition $P$ of $N$ is said to respect $(N, D)$ when the following five properties hold:

$$
\begin{aligned}
& \text { P1 If }(i, j) \in D \text {, then }\left[P_{i} \neq\{i\}\right] \Longrightarrow\left[j \in P_{i}\right] . \\
& \text { P2 If } j \in A(i) \text {, then }\left[P_{i} \backslash\left(\widehat{S}_{D}(i) \cup i\right) \neq \emptyset\right] \Longrightarrow\left[j \in P_{i}\right] . \\
& \text { P3 If } l(i)=l(j) \text {, then }\left[S_{D}(i) \neq \emptyset \text { and } P_{i}=\{i\}\right] \Longrightarrow\left[P_{j}=\{j\}\right] . \\
& \text { P4 If }(i, j) \in D \text {, then }\left[P_{i}=\{i\}\right] \Longrightarrow\left[P_{j} \subseteq \widehat{S}_{D}(i)\right] . \\
& \text { P5 If } l(i)=l(j), A(i) \backslash i \neq \emptyset \text {, and } A(j) \backslash j \neq \emptyset \text {, then }\left[P_{i} \subseteq\left(\widehat{S}_{D}(i) \cup i\right)\right] \Longleftrightarrow\left[P_{j} \subseteq\left(\widehat{S}_{D}(j) \cup j\right)\right] .
\end{aligned}
$$

The first property, P1, states that if within the partition, a player depends on some other player in the sense that she is in the same union with this other player, then she must be in the same union of the partition as all other members of her team. This holds no matter whether within the partition, a player depends on another player who is in her team or on a player who is not. P2 states that if within the partition, a player $i$ depends on some player who is not in 
her team, then all $i$ 's siblings must belong to the same union as $i$. P3 requires that if a player is a singleton in the partition while having at least one successor in the directed tree, then any other player with the same rank should also be a singleton. According to P4, when a player is a singleton in the partition, then none of the members of her team depends on a player who does not belong to this team. Finally, P5 states that for any two players with the same rank who each have one sibling at least, either within the partition they only depend on their team or they depend on a player who is not in her team.

Finally, we characterize the mappings that convert any directed tree $(N, D) \in \mathcal{D}$ into a levels structure $(N, \underline{B})$, where any partition $P \in \underline{B} \operatorname{respects}(N, D)$, i.e., it satisfies the five properties P1-P5. Recall that $\left(N, B^{D}\right)=\mathcal{H}(N, D)$ denotes the levels structure defined by Eqs. (3.2)-(3.4). Theorem 3.2. Consider a directed tree $(N, D) \in \mathcal{D}$. Then, a proper partition $P$ of $N$ respects $(N, D)$ if and only if $P \in B^{D}$. Moreover, the five properties of Definition 3.1 are independent.

Note that the mapping assigning the trivial partition $\{N\}$ with $N$ as its unique element to every directed tree also satisfies the five properties. Since some properties become meaningless for this partition, we have only considered proper partitions in the theorem. The next corollary follows immediately from Theorem 3.2.

Corollary 3.1. Any mapping $\mathcal{I}: \mathcal{D} \rightarrow \mathcal{L}$, such that every partition in $\mathcal{I}(N, D)$ respects $(N, D)$ and has a maximal number of different partitions, can be built from the partitions in $\left(N, B^{D}\right)$.

To sum up, in this section we have focused on a particular way to map a directed tree into a levels structure, namely that every partition of the levels structure respects the tree in the sense of Definition 3.1. The properties P1-P5 and Theorem 3.2 imply that in such a levels structure, a player that is higher in the hierarchy is more independent from the other players in the collection of nested partitions, in the sense that such a player becomes a singleton coalition at a lowerranked partition in the levels structure than another player who is lower in the hierarchy. ${ }^{9}$ While the proposed mapping between the set of directed trees and the set of levels structures has value in its own right, due to the way it is constructed and because of the properties it satisfies, it will turn out that the solutions for games with hierarchical structure to be introduced hereafter will add even more to its relevance.

\footnotetext{
${ }^{9}$ We stress that more independent does not mean better off, not even for monotone games. An elaboration of this argument can be found in Álvarez-Mozos et al. (2015).
} 


\section{A New Solution for Games with Hierarchical Structure}

We are now ready to introduce and axiomatize a new type of solution for the class of games with hierarchical structure, $\mathcal{G D}$. For each solution of this type, we follow a two-stage procedure. First, the hierarchical structure $(N, D)$ is transformed into a levels structure $(N, \underline{B})$, with $\underline{B}$ being a subset of the collection $B^{D}$ of partitions generated by the conversion mapping $\mathcal{H}$. Second, the Shapley Levels Value is applied to the game with levels structure obtained in the first stage. The first solution of this type that we propose is obtained by taking the levels structure $\left(N, B^{D}\right)=\mathcal{H}(N, D)$, i.e., by considering the entire collection of partitions that satisfy the five properties of Theorem 3.2.

Definition 4.1. The solution $\varphi^{S L}$ on the class of games with hierarchical structure $\mathcal{G D}$ is the solution given by

$$
\varphi^{S L}(N, v, D)=S h^{L}\left(N, v, B^{D}\right), \text { with }(N, v, D) \in \mathcal{G D}
$$

By definition, the first level of $\left(N, B^{D}\right)$ consists of two unions: the root player as a singleton and the set of all other players. Consequently, because $\varphi^{S L}$ is obtained by applying $S h^{L}$ to $\left(N, B^{D}\right)$, besides receiving her singleton worth (dividend), the root player earns half the dividends of all coalitions she belongs to. Any other player $i$ with at least one subordinate belongs to the same union as all her subordinates for any partition $B_{r, l}^{D}$ such that $(r, l) \leq_{l e x}(l(i), 1)$, with $i$ 's team being a union in $B_{l(i), 2}^{D} \cdot{ }^{10}$ At the next level, namely at the level corresponding to $B_{l(i)+1,1}^{D}$, player $i$ then becomes a singleton and all her subordinates form a union by themselves. Hence, each player obtains a share equal to the sum of all shares of all her subordinates in the dividends of any coalition to which she belongs together with at least one of her subordinates. We illustrate the new solution with an example.

Example 4.1. Consider $\left(N, u_{N}, D\right) \in \mathcal{G D}$, with $(N, D)$ the hierarchy given in Example 3.1. Then,

$$
\varphi^{S L}\left(N, u_{N}, D\right)=\left(\frac{1}{2}, \frac{1}{8}, \frac{1}{8}, \frac{1}{16}, \frac{1}{32}, \frac{1}{8}, \frac{1}{64}, \frac{1}{64}\right) .
$$

Note that the root of the tree obtains half the worth of the grand coalition. Then, the proposed value assigns half the remainder to Player 2 and her subordinates, and half the remainder to Player 3 and her subordinates, and so on, until the last level of the levels structure is reached.

\footnotetext{
${ }^{10}$ For $(r, l),(r, l) \in \mathbb{N} \times \mathbb{N}$, we write $(r, l) \leq$ lex $\left(r^{\prime}, l^{\prime}\right)$ if either $r<r^{\prime}$ or $r=r^{\prime}$ and $l \leq l^{\prime}$.
} 
In the following, we provide a set of properties (or axioms) that will characterize the value $\varphi^{S L}$ on the class $\mathcal{G} \mathcal{D}$. The first three axioms are natural generalizations of the classical efficiency, additivity, and null player properties.

EFF A solution $f$ on $\mathcal{G D}$ satisfies Efficiency if for every $(N, v, D) \in \mathcal{G D}$,

$$
\sum_{i \in N} f_{i}(N, v, D)=v(N)
$$

ADD A solution $f$ on $\mathcal{G D}$ satisfies Additivity if for every $(N, v, D),(N, w, D) \in \mathcal{G D},{ }^{11}$

$$
f(N, v+w, D)=f(N, v, D)+f(N, w, D) .
$$

NPP A solution $f$ on $\mathcal{G D}$ satisfies the Null Player Property if for every $(N, v, D) \in \mathcal{G D}$ and $i \in N$ a null player in $(N, v)$,

$$
f_{i}(N, v, D)=0 .
$$

While EFF and ADD are standard, the use of NPP in our framework needs some justification. We start by noting that we are not the first to use NPP within a setting of restricted cooperation. Faigle and Kern (1992), in particular, included such a property in their axiomatization of the Precedence Shapley Value. More recently, Fiestras-Janeiro et al. (2016) have also considered NPP to characterize the Precedence Shapley Value in a special class of games under precedence constraints. ${ }^{12}$ NPP establishes a weak necessary condition for a player to earn a non-zero payoff: her contributions to all other coalitions of players, including the empty coalition, cannot all be zero. Hence, an important feature of NPP is that it is independent of the hierarchy. We impose NPP throughout the paper.

In contrast with the above two solutions, the Permission Value does not satisfy NPP. ${ }^{13}$ It does however satisfy the Necessary Player Property, which is defined below.

\footnotetext{
${ }^{11}$ Given two games $(N, v)$ and $(N, w)$, the game $(N, v+w)$ is defined by $(v+w)(S)=v(S)+w(S)$ for all $S \subseteq N$.

${ }^{12}$ Fiestras-Janeiro et al. (2016) consider hierarchical structures represented by an ordered partition of the player set, i.e. a partition with a linear order on the elements of the partition. In that context, they call this solution the Hierarchical Value.

${ }^{13}$ In van den Brink and Gilles (1996) and van den Brink (1997), the weaker Inessential Player Property is used to axiomatize the Permission Value by requiring that a null player in $(N, v)$ has to earn a zero payoff if her subordinates in $(N, D)$ are all also null players in $(N, v)$.
} 
NEPP A solution $f$ on $\mathcal{G D}$ satisfies the Necessary Player Property if for every $(N, v, D) \in \mathcal{G D}$ with $(N, v) \in \mathcal{G}_{M}, i \in N$ a necessary player in $(N, v)$, and $j \in N \backslash\{i\}$,

$$
f_{i}(N, v, D) \geq f_{j}(N, v, D)
$$

The above property requires that in a monotone game, a necessary player has to earn at least as much as any other player, irrespective of her position in the hierarchy. As mentioned in the Introduction, requiring EFF, ADD, NPP, and NEPP yields the Shapley Value of the game and thus completely ignores the hierarchy. Therefore, the combination of these four properties is not particularly appealing for games with hierarchical structure. To circumvent this limitation, we shall consider a collection of properties that result from weakening NEPP. Each of these properties compares a player's payoff to the payoff of a different set of players with whom she is related in the hierarchy in some particular way, viz. her siblings, her team, her successors' team, or her siblings' successors. It is important to point out that all these properties apply in the class of monotone games only, so they do not impose any requirement for non-monotone games.

The first weakening of NEPP requires that a necessary player has to earn at least as much as any of her siblings if they all are at the bottom of the tree. Since any two such players are symmetric in the hierarchy, any of them will be in a stronger position overall if she is necessary in the game. This implies, in particular, that two siblings who are necessary in the game and have no successors have to receive the same payoff, and thus this first weakening of NEPP can be seen as a weak symmetry property.

NSP A solution $f$ on $\mathcal{G D}$ satisfies the Necessary Sibling Property if for every $(N, v, D) \in \mathcal{G D}$ with $(N, v) \in \mathcal{G}_{M}$, if two players $i, j \in N$ are such that $S_{D}(i)=S_{D}(j)=\emptyset, i \in A(j)$, and if $i$ is a necessary player in $(N, v)$,

$$
f_{i}(N, v, D) \geq f_{j}(N, v, D)
$$

Instead of comparing the payoffs of siblings, who are related to each other in the hierarchy from a horizontal perspective, we now explore a vertical perspective by relating a player's payoff to the total payoff of all her subordinates, provided that at least one of them is a necessary player. 
SUB A solution $f$ on $\mathcal{G D}$ satisfies the Superior Upper Bound if for every $(N, v, D) \in \mathcal{G D}$ with $(N, v) \in \mathcal{G}_{M}$, if $j \in \widehat{S}_{D}(i)$ is necessary in $(N, v)$ for some $i \in N$,

$$
f_{i}(N, v, D) \leq \sum_{h \in \widehat{S}_{D}(i)} f_{h}(N, v, D)
$$

The above property requires that in a monotone game a player, say $i$, cannot earn more than the total payoff of her subordinates when at least one them, say $j$, is a necessary player. Note that NEPP would require that $j$ earns at least as much as $i$. As a consequence, SUB is weaker than NEPP, provided that all payoffs are non-negative. Having introduced SUB, a natural counterpart is to require that a necessary player has to earn at least as much as all her subordinates combined.

SLB A solution $f$ on $\mathcal{G D}$ satisfies the Superior Lower Bound if for every $(N, v, D) \in \mathcal{G D}$ with $(N, v) \in \mathcal{G}_{M}$, if $i \in N$ is necessary in $(N, v)$,

$$
f_{i}(N, v, D) \geq \sum_{j \in \widehat{S}_{D}(i)} f_{j}(N, v, D) .
$$

Note that SUB and SLB are not merely obtained from each other by reversing the inequalities: the necessary players are different. We further point out that SLB, as well as other similar properties introduced in the next section, is not a weakening of NEPP. In fact, the two properties are not logically related. For the sake of exposition, we have named the properties from the point of view of the superior, whether she is a necessary player or not.

Finally, we introduce a property that suggests how a solution should behave with respect to certain changes in the hierarchical structure. More specifically, the property below states that merging all players of one team cannot not affect the payoffs of the other players. Merging player $j$ with the rest of her team $\widehat{S}_{D}(j)$ in game $(N, v, D)$ yields a new game $\left(N \backslash \widehat{S}_{D}(j), v^{j}, D_{N \backslash \widehat{S}_{D}(j)}\right)$, with $v^{j}$ defined for every $T \subseteq N \backslash \widehat{S}_{D}(j)$ by

$$
v^{j}(T)= \begin{cases}v(T) & \text { if } j \notin T, \\ v\left(T \cup \widehat{S}_{D}(j)\right) & \text { if } j \in T .\end{cases}
$$

When a team merges as just described, the role of the other players in the hierarchy does not change. The only difference is that as a result of the merger, the latter players only have to deal with the top player of the merged team, instead of having to deal with each of the players 
of such team. The next property requires that as long as productivities remain unaffected by the merger of a team, so should the payoffs of the players who do not take part in the merger.

IMT A solution $f$ on $\mathcal{G D}$ satisfies Independence of Merging Teams if for every $(N, v, D) \in \mathcal{G D}$, $j \in N$, and $i \in N \backslash\left(\widehat{S}_{D}(j) \cup j\right)$,

$$
f_{i}(N, v, D)=f_{i}\left(N \backslash \widehat{S}_{D}(j), v^{j}, D_{N \backslash \widehat{S}_{D}(j)}\right) .
$$

The above property may be especially appealing in certain real-world allocation problems. For instance, it can be interpreted as an autonomy property for regions in a country or for departments in an organization such as a firm or a university. Whatever the specific setting, IMT provides different units with the freedom to reorganize themselves without affecting the entire payoff structure. In combination with EFF, this independence property further implies that when all players in a team merge - and delegate their role to the team's boss - , the boss's payoff in the reduced hierarchy has to be equal to the sum of the payoffs of all members of the team in the original hierarchy. As a consequence, under IMT, there exist no incentives for a team to merge as far as the team's aggregate payoff is concerned.

It turns out that the properties introduced throughout the section characterize the solution $\varphi^{S L}$ on the class of games with hierarchical structure.

Theorem I. A solution $f$ on $\mathcal{G D}$ satisfies $\mathrm{EFF}$, ADD, NPP, IMT, NSP, SLB, and SUB, if and only if $f=\varphi^{S L}$. Moreover, the seven properties are independent.

In the next section, we consider different versions of upper and lower bound properties that lead to two alternative solutions for games with hierarchical structure.

\section{Two Alternative Solutions}

The solution $\varphi^{S L}$ proposed in the previous section uses the entire levels structure of the conversion mapping defined in Section 3. That is to say, all the information about the tree preserved by the conversion mapping is used. In the present section, we propose two alternative solutions for games with hierarchical structure. Both alternatives are based on the same procedure used to construct $\varphi^{S L}$, yet with some changes. First, the hierarchical structure $(N, D)$ is transformed 
into a levels structure $(N, \underline{B})$, with $\underline{B}$ now being a subset of the collection $B^{D}$ of partitions generated by the conversion mapping $\mathcal{H}$ instead of the entire collection. Second, the Shapley Levels Value is applied to the game with levels structure obtained in the first stage.

\subsection{A first alternative solution based on the team levels}

For the first alternative solution, we consider the levels structure $\left(N, \bar{B}^{D}\right)$ given by $\bar{B}^{D}=$ $\left(\bar{B}_{1}^{D}, \bar{B}_{2}^{D}, \ldots, \bar{B}_{l(D)}^{D}\right)$, where

$$
\bar{B}_{k}^{D}=B_{k, 2}^{D} \text { for } k \in\{1, \ldots, l(D)\},
$$

with $B_{k, 2}^{D}$ as given by Eq. (3.4). Compared with the original levels structure $\left(N, B^{D}\right)$, we now only consider all partitions $B_{k, 2}^{D}$, with $k \in\{1, \ldots, l(D)\} .{ }^{14}$ That is, we admit all the team levels (even levels) and disregard all the siblings levels (odd levels). Since each element in a partition is either a singleton or a team, this approach builds on the assumption that teams are the relevant organizational units of production. To every $(N, v, D) \in \mathcal{G D}$, the first alternative solution assigns the Shapley Levels Value of the game with levels structure $\left(N, v, \bar{B}^{D}\right)$.

Definition 5.1. The solution $\bar{\varphi}^{S L}$ on the class of games with hierarchical structure $\mathcal{G D}$ is the solution given by

$$
\bar{\varphi}^{S L}(N, v, D)=S h^{L}\left(N, v, \bar{B}^{D}\right) \text {, with }(N, v, D) \in \mathcal{G D}
$$

We illustrate the above definition by an example.

Example 5.1. Consider $\left(N, u_{N}, D\right) \in \mathcal{G D}$, with $(N, D)$ being the hierarchy given in Example 3.1. Then, the level structure $\left(N, \bar{B}^{D}\right)$ consists of the three even levels. Compared to the level structure used for solution $\varphi^{S L}$, the first level's partition of $\left(N, B^{D}\right)$ consists of the top player as a singleton and the teams of each of her two successors. This yields the payoff vector given by

$$
\bar{\varphi}^{S L}\left(N, u_{N}, D\right)=\left(\frac{1}{3}, \frac{1}{9}, \frac{1}{6}, \frac{1}{9}, \frac{1}{27}, \frac{1}{6}, \frac{1}{27}, \frac{1}{27}\right) .
$$

The example above reveals that $\bar{\varphi}^{S L}$ does not satisfy SLB, since player 1 earns less than her subordinates jointly, despite the fact that she is necessary in $\left(N, u_{N}\right)$. The reason is that in the

\footnotetext{
${ }^{14}$ Note that $\bar{B}_{l(D)}^{D}$ is the trivial partition of singletons.
} 
less refined levels structure $\left(N, \bar{B}^{D}\right)$, the subordinates of a player, say $i$, are grouped in different teams of $i$ 's successors right at the first level where player $i$ is separated from her subordinates.

For necessary players and when the game is monotone, properties SLB and SUB (see Section 4) establish a relation between a player's payoff and the sum of the payoffs of all other players in her team or, equivalently, the sum of the payoffs of all her successors' teams. Indeed, we stress that $\widehat{S}_{D}(i)=\bigcup_{j \in S_{D}(i)}\left[\{j\} \cup \widehat{S}_{D}(j)\right]$ for all $i \in N$. Thus, all these teams, i.e. $\left[\{j\} \cup \widehat{S}_{D}(j)\right]$ with $j \in S_{D}(i)$, are considered at once. Nevertheless, under the assumption that teams are autonomous, it might instead be appealing to relate the payoff of a player to the total payoff of the players in each of her successors' teams separately. We do so in the following two properties, with the so-called team upper bound property being again a weakening of NEPP-provided that payoffs are non-negative - and the team lower bound property being its counterpart.

TUB A solution on $\mathcal{G D}, f$, satisfies the Team Upper Bound if for every $(N, v, D) \in \mathcal{G D}$ with $(N, v) \in \mathcal{G}_{M}$, if $j \in \widehat{S}_{D}(i)$ is necessary in $(N, v)$ for some $i \in N$ and $h \in S_{D}(i) \cap$ $\left(\widehat{P}_{D}(j) \cup j\right),{ }^{15}$

$$
f_{i}(N, v, D) \leq \sum_{k \in \widehat{S}_{D}(h) \cup h} f_{k}(N, v, D) .
$$

TLB A solution on $\mathcal{G D}, f$, satisfies the Team Lower Bound if for every $(N, v, D) \in \mathcal{G D}$ with $(N, v) \in \mathcal{G}_{M}$, if $i \in N$ is necessary in $(N, v)$ and $j \in S_{D}(i)$,

$$
f_{i}(N, v, D) \geq \sum_{k \in \widehat{S}_{D}(j) \cup j} f_{k}(N, v, D) .
$$

It is easy to verify that SLB $\Rightarrow$ TLB and SUB $\Leftarrow$ TUB. Substituting SLB and SUB with TLB and TUB in Theorem I singles out solution $\bar{\varphi}^{S L}$.

Theorem II. A solution $f$ on $\mathcal{G D}$ satisfies EFF, ADD, NPP, IMT, NSP, TLB, and TUB if and only if $f=\bar{\varphi}^{S L}$. Moreover, the seven properties are independent.

In Theorems I and II, we have used the superior and team upper- and lower-bound properties to characterize the solution $\varphi^{S L}$ that uses the most refined levels structure and the solution $\bar{\varphi}^{S L}$ that uses only the team levels (even levels), respectively. An ensuing question is what solution results from using only the sibling levels (odd levels). We turn to that next.

\footnotetext{
${ }^{15}$ Note that $h$ is uniquely determined.
} 


\subsection{A second alternative solution based on the sibling levels}

As just mentioned, a further solution for games with hierarchical structure can be obtained by keeping from $B^{D}$ only the partitions $B_{k, 1}^{D}$, with the caveat that we have to add the trivial partition of singletons $B_{l(D), 2}^{D}$ in order for such a sequence of partitions to formally be a levels structure. If we do so, we obtain the levels structure $\left(N, \widetilde{B}^{D}\right)$ with $\widetilde{B}^{D}=\left(\widetilde{B}_{1}^{D}, \widetilde{B}_{l(D)}^{D}, \widetilde{B}_{l(D)+1}^{D}\right)$, where $\widetilde{B}_{k}^{D}=B_{k, 1}^{D}$ for $k \in\{1, \ldots, l(D)\}$ and $\widetilde{B}_{l(D)+1}^{D}=B_{l(D), 2}^{D}=\{\{i\}: i \in N\}$, which induces the following definition:

Definition 5.2. The solution $\widetilde{\varphi}^{S L}$ on the class of games with hierarchical structure $\mathcal{G D}$ is the solution given by

$$
\widetilde{\varphi}^{S L}(N, v, D)=S h^{L}\left(N, v, \widetilde{B}^{D}\right) \text {, with }(N, v, D) \in \mathcal{G D} \text {. }
$$

There is an obvious sense in which this third solution can be considered to be the complement of $\bar{\varphi}^{S L}$. The reason is that except for the last level, both solutions use disjoint subsets of the information contained in the levels structure obtained by applying $\mathcal{H}$ to the original hierarchical structure. As for the first and second solutions, we illustrate the definition of the third solution by our example.

Example 5.2. Consider again $\left(N, u_{N}, D\right) \in \mathcal{G D}$, with $(N, D)$ being the hierarchy given in Example 3.1. Then, the level structure $\left(N, \widetilde{B}^{D}\right)$ consists of the three odd levels plus the level $B_{3,2}^{D}$ of singletons. In that case, the first level consists of two unions, the top player being a singleton union and all other players, i.e., all her successors together with all their team members, being in the other union. Applying the Shapley Levels Value yields the payoff vector

$$
\widetilde{\varphi}^{S L}\left(N, u_{N}, D\right)=\left(\frac{1}{2}, \frac{1}{8}, \frac{1}{8}, \frac{1}{24}, \frac{1}{24}, \frac{1}{8}, \frac{1}{48}, \frac{1}{48}\right) .
$$

From an inspection of the payoffs in the example above, it follows that $\widetilde{\varphi}^{S L}$ does not satisfy TUB. Take $i=2$ and $j=h=4$, for instance. Then, $\{4\}$ is a team that contains a necessary player in $\left(N, u_{N}\right)$, but the payoff to player 2 is greater than the payoff to player 4 . It is also straightforward to see that $\widetilde{\varphi}^{S L}$ does not satisfy IMT. Take for instance $i=2$ and $j=3$. Then, $i$ 's payoff becomes $\frac{1}{6}$ when $j$ merges with her team. Interestingly, however, $\widetilde{\varphi}^{S L}$ does satisfy stronger versions of NSP, SUB, and SLB, all of which are introduced next. 
First, we consider a property that relates the payoff of any necessary player to the payoffs of her siblings in monotone games. Unlike NSP, the requirement on the payoffs does not only apply to siblings at the bottom of the hierarchy, but also to siblings that may have successors.

SNSP A solution on $\mathcal{G D}, f$, satisfies the Strong Necessary Sibling Property if for every $(N, v, D) \in$ $\mathcal{G D}$ with $(N, v) \in \mathcal{G}_{M}$, if $i \in N$ is necessary in $(N, v)$ and $j \in A(i)$,

$$
f_{i}(N, v, D) \geq f_{j}(N, v, D) .
$$

Note that SNSP $\Rightarrow$ NSP, i.e., SNSP is stronger than NSP. Nevertheless, SNSP is still weaker than NEPP.

Second, we consider two properties that relate the payoff of a player, say $i$, to the total payoff of the subordinates of one of $i$ 's siblings, provided that either there is a necessary player amongst the latter or $i$ herself is a necessary player, respectively.

SSUB A solution on $\mathcal{G D}, f$, satisfies the Strong Superior Upper Bound if for every $(N, v, D) \in \mathcal{G D}$ with $(N, v) \in \mathcal{G}_{M}$ and every $i \in N$, if $h \in A(i)$ and some $j \in \widehat{S}_{D}(h)$ is necessary in $(N, v)$,

$$
f_{i}(N, v, D) \leq \sum_{p \in \widehat{S}_{D}(h)} f_{p}(N, v, D) .
$$

SSLB A solution on $\mathcal{G D}, f$, satisfies the Strong Superior Lower Bound if for every $(N, v, D) \in \mathcal{G D}$ with $(N, v) \in \mathcal{G}_{M}$, if $i \in N$ is necessary in $(N, v)$ and $j \in A(i)$,

$$
f_{i}(N, v, D) \geq \sum_{h \in \widehat{S}_{D}(j)} f_{h}(N, v, D) .
$$

Provided that payoffs are non-negative, SSUB is a weakening of NEPP. Moreover, because $i \in A(i)$, SSLB $\Rightarrow$ SLB and SSUB $\Rightarrow$ SUB. While $\widetilde{\varphi}^{S L}$ satisfies SSUB and SSLB-see Theorem III below-, it does not satisfy IMT, as we have seen in Example 5.2. This implies that a property requiring a connection between the payoffs of two groups of players who are only related in the hierarchy at a higher level is needed to characterize $\widetilde{\varphi}^{S L}$. For both $\varphi^{S L}$ and $\bar{\varphi}^{S L}$, IMT plays such a role. In the case of $\widetilde{\varphi}^{S L}$, by contrast, we consider a property that relates the payoff of the subordinates of two players when one of the former is necessary in the game. 
NOP A solution on $\mathcal{G D}, f$, satisfies the Necessary Offspring Property if for every $(N, v, D) \in \mathcal{G D}$ and every $i \in N$ such that $\widehat{S}_{D}(i)$ contains necessary players, it holds that for every $j \in A(i)$,

$$
\sum_{h \in \widehat{S}_{D}(i)} f_{h}(N, v, D) \geq \sum_{h \in \widehat{S}_{D}(j)} f_{h}(N, v, D)
$$

The above property can be seen as a variation of NSP and SNSP in the following sense: instead of comparing the payoffs of two siblings when one of them is a necessary player in the game, as NSP and SNSP do, NOP relates the total payoff of the two sets of subordinates of two siblings by requiring that the total payoff to one of the sets is at least as much as the total payoff to the other one if the former contains a necessary player. Requiring this might be reasonable in situations where players identify themselves with their (local) boss, and so the relation in the hierarchy between the two siblings translates into a relation between the two sets of subordinates.

Theorem III. A solution $f$ on $\mathcal{G D}$ satisfies $\mathrm{EFF}, \mathrm{ADD}$, NPP, SNSP, SSLB, SSUB, and NOP if and only if $f=\widetilde{\varphi}^{S L}$. Moreover, the seven properties are independent.

That is, if in Theorem I we replace NSP, SUB, and SLB by the stronger properties SNSP, SSUB, and SSLB, and IMT by NOP, we single out solution $\widetilde{\varphi}^{S L}$ instead of $\varphi^{S L}$.

\section{Properties and Solutions: Assessment and Comparison}

The goal of this section is two-fold. First, we examine at length the properties for solutions of games with hierarchical structure that we have introduced throughout the paper. Second, we compare our three new solutions to the best-known solutions that have been proposed so far in the literature.

\subsection{Assessment of the properties}

As we have already mentioned, the Necessary Player Property, NEPP, is too demanding a condition in our setting. This follows from the fact that when such a property is combined with EFF, ADD, and NPP, it leads to a solution that ignores the hierarchical structure. Throughout the paper, we have explored several ways to weaken NEPP, namely NSP, SUB, TUB, SNSP, and SSUB, each variant then leading to a different solution (in combination with other axioms). Because of 
the importance that such properties have in our analysis, we start this section by showing the logical relations between them and NEPP in a diagram.

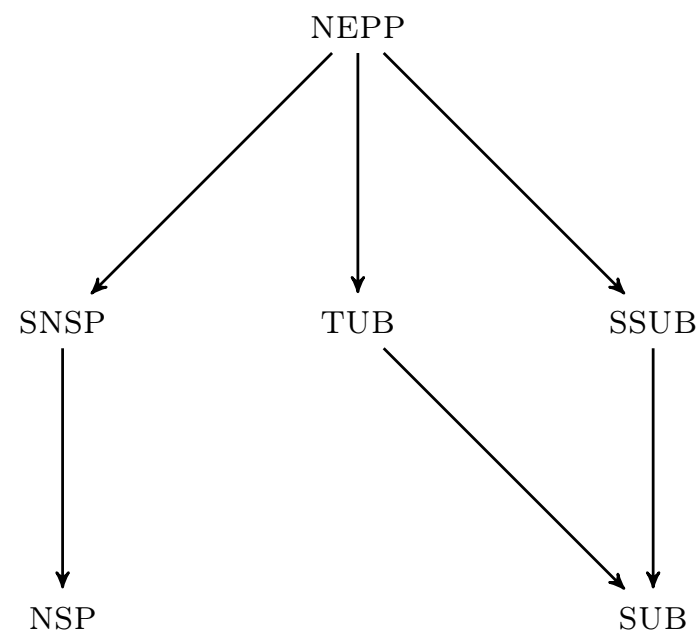

Figure 1: The logical relations between the Necessary Player Property (NEPP) and its weakenings (provided that payoffs are non-negative).

Having illustrated the logical relations between NEPP and its weakenings, we now proceed with the rest of the section. Beyond methodological differences, our three solutions diverge significantly from an axiomatic viewpoint. This has been delineated in the previous section based on the characterizations of the three solutions by means of certain properties, including some of the weakenings of NEPP just mentioned. Since many of these properties are new in the literature, their use has to be justified. ${ }^{16}$

First, we focus on NSP. This weakening of NEPP is satisfied by all three solutions. Because it can be seen as a very weak symmetry axiom, it is then quite reasonable for many real-world applications. Indeed, NSP only requires that for two players without successors who have the same boss, and thus have identical positions in the hierarchy, their relative payoff has to be fully determined by their marginal contributions in the game. An extremely weak implementation of such a condition is to require that if one of the players is necessary in the game, she has to earn at least as much as the other player.

Second, a central role in the characterizations of the new solutions is played by the so-called

\footnotetext{
${ }^{16}$ Most of the properties that we discuss below are only required for monotone games. However, to facilitate the discussion, we shall not mention this feature again.
} 
upper bound (SUB, TUB, and SSUB) and lower bound (SLB, TLB, and SSLB) properties. All these axioms relate a player's payoff to the payoff of certain other players with whom she is related in the hierarchy. On the one hand, the upper bound properties arise from weakening NEPP, provided that payoffs are non-negative. On the other hand, the corresponding lower bound properties are natural counterparts, and it seems reasonable to discuss them in pairs, namely $\mathrm{SUB} / \mathrm{SLB}, \mathrm{TUB} / \mathrm{TLB}$, and SSUB/SSLB.

We start by reflecting on SUB and SLB. These two properties require a comparison to be made between a player's payoff and the total payoff of her subordinates. Doing so seems particularly reasonable in a firm, given the fact that SUB relaxes the assumption often made in the literature that a manager's wage should not be more than the sum of all her (direct) successors's wages - see e.g. Williamson (1967). SUB weakens this in two ways. First, provided that payoffs are non-negative, it relaxes the upper bound by considering the sum of the wages of all her subordinates instead of that of her successors only. Second, it focuses on the case where at least one subordinate is necessary. In turn, a motivation for SLB is as follows: Suppose that a manager without whom the firm cannot produce any worth, i.e. a necessary player, has only one subordinate. Then, it is reasonable to demand that the manager earn at least as much as the subordinate. A natural generalization of this property when the manager has more subordinates is thus to require that the manager has to earn at least as much as the sum of the payoffs of all her subordinates. This is precisely what SLB demands. Furthermore, we note that the latter property is related to some extent to the axiom of structural monotonicity (SM), although both axioms are not logically related. Property SM is used in van den Brink and Gilles (1996) to axiomatize the Permission Value, and it requires that a player has to earn at least as much as each of her successors. On the one hand, provided that payoffs are non-negative, SLB has a stronger flavor than SM in that it considers the total payoff of all the subordinates of a player instead of the payoff of a successor. On the other hand, SLB has a weaker flavor than SM in that it requires the inequality to hold if and only if the manager is necessary in the game.

We now focus on TUB and TLB. On the one hand, because SLB $\Rightarrow$ TLB, the latter property should be acceptable if SLB is so. Moreover, likewise SLB, TLB can also be related to SM. On the other hand, as an alternative to SUB, TUB takes the team of one of the player's successors as reference instead of taking the set of all subordinates of the player, as SUB does. For organizations 
where teams act more or less independently, TUB seems thus more reasonable. ${ }^{17}$

Next, consider SSUB and SSLB. These two properties share some features of NSP, for they compare a player's payoff with that of a coalition of players who are all related to her siblings. Moreover, like TLB and TUB, SSUB and SSLB consider teams instead of individual players. Requiring both SSUB and SSLB, in particular, can be useful when comparing branches of different size. Indeed, suppose that player $i$ is the head of some department-namely, the branch below her-, and that one of her siblings is the head of a much bigger department. According to NSP, if player $i$ and her sibling both had no successors, $i$ would then earn at least as much as her sibling, provided that she is necessary in the game. When both $i$ and her sibling have successors, however, NSP has no bite. In such a situation, player $i$ could instead claim at least as much as all her siblings' subordinates combined. This is precisely what SSLB demands. Finally, SSUB can further be justified as a natural counterpart of SSLB, analogously as SUB and TUB are the natural counterparts of SLB and TLB, respectively.

Third, we discuss SNSP and NOP. On the one hand, SNSP strengthens NSP by demanding that any necessary player in the game has to receive at least as much as any of her siblings. Yet, requiring so is still less stringent than NEPP. A rationale for the application of SNSP is to assume that siblings should be treated equitably as far as their position in the hierarchy is concerned, no matter how their teams are organized. In particular, their relative payoff should be determined solely by their marginal contributions in the game. SNSP implements such a condition. On the other hand, NOP fills the gap needed to complete the characterization of $\widetilde{\varphi}^{S L}$, which has been primarily motivated from a methodological viewpoint as the complement of $\bar{\varphi}^{S L}$. Precisely because of this, however, NOP unravels which coalitions of players are to be compared in terms of their aggregate payoffs: teams in the case of solution $\bar{\varphi}^{S L}$ (recall TUB and TLB) as opposed to teams without their boss in the case of solution $\widetilde{\varphi}^{S L}$ (recall SSUB, SSLB, and NOP).

Fourth and last, IMT can be discussed beyond the comments made in Section 4. This property requires that when a player and all her subordinates merge, payoffs of all other players cannot

\footnotetext{
${ }^{17}$ We stress that the difference between $\varphi^{S L}$ and $\bar{\varphi}^{S L}$ boils down to choosing either the pair SLB/SUB or the pair TLB/TUB, respectively. The decision about which pair to choose should depend on the application considered. For instance, if we were to interpret a hierarchical structure as a model for the different layers of public administration, SLB/SUB would probably be a better (worse) description than TLB/TUB of a centralized (decentralized) budget distribution.
} 
change. Similar vertical and horizontal merge (split) neutrality axioms have been considered in van den Brink (2010). Instead of requiring that a merger cannot affect the players who are not involved, however, these axioms require that after the merger, the payoff of the resulting merged player has to be equal to the sum of all merging players. Hence, rather than stipulating an independence condition, as IMT does, these neutrality axioms require a type of payoff stability, so that players have no incentive to either merge or split. In the literature on communication graph games, so-called independence axioms have been considered, which do not consider a merger in the structure of the graph, but a merger in the game. Similarly to IMT, those axioms require that the payoff of players who are not involved in the merger has to remain unchanged. Such an independence axiom on the class of unanimity games is used in Mishra and Talman (2010) to characterize the Average Tree Solution for communication graph games.

\subsection{Comparison with other values}

We now turn to the issue of which of the new properties are satisfied by other solutions from the literature. Doing so will enable us to examine our three solutions more thoroughly from a comparative, axiomatic viewpoint. We focus on five well-known solutions, which we now describe informally for the sake of completeness.

First, the Precedence Shapley Value (PSV, Faigle and Kern, 1992) is obtained as the average of the marginal vectors for which successors enter before their predecessors in the directed tree. Second, the Permission Value (PV, van den Brink and Gilles, 1996) is obtained as the Shapley Value of the permission restricted game. To every coalition, this game assigns the worth of its largest feasible subset in the original game. A set of players is feasible if for every player in the set, all her predecessors are also in the set. Third, to every player $i$, the Hierarchical Outcome (HO, Demange, 2004) assigns the worth of her team, $v\left(\{i\} \cup \widehat{S}_{D}(i)\right.$ ), minus the sum of the worths of the teams of all her successors, $\sum_{j \in S_{D}(i)} v\left(\{j\} \cup \widehat{S}_{D}(j)\right) .{ }^{18}$ Fourth, the Average Tree Permission Value (ATPV, van den Brink et al., 2015) applies the Average Tree Value defined in Herings et al. (2005) to an associated undirected graph game, where the game is the permission restricted game and the graph is the undirected graph obtained from the hierarchical structure

\footnotetext{
${ }^{18}$ The Hierarchical Outcome and the Permission Value are compared with two other values in van den Brink et al. (2016).
} 
by ignoring the orientation of the arcs. ${ }^{19}$ Finally, for the sake of completeness, we also consider the solution $S h$, which assigns to every game with hierarchical structure the Shapley Value of the game, and thus ignores the hierarchical structure.

It can be easily verified that the above five solutions, as well as our three new solutions, satisfy EFF and ADD. For the remaining properties, we present Table 1.

\begin{tabular}{|l|c||c|c||c|c|c||c|c|c||c|c|}
\cline { 2 - 11 } \multicolumn{1}{c|}{} & NPP & NSP & SNSP & SUB & TUB & SSUB & SLB & TLB & SSLB & IMT & NOP \\
\hline$\varphi^{S L}$ & $*$ & $*$ & - & $*$ & - & - & $*$ & + & - & $*$ & - \\
\hline $\bar{\varphi}^{S L}$ & $*$ & $*$ & - & + & $*$ & - & - & $*$ & - & $*$ & - \\
\hline$\widetilde{\varphi}^{S L}$ & $*$ & + & $*$ & + & - & $*$ & + & + & $*$ & - & $*$ \\
\hline$S h$ & + & + & + & + & - & + & - & + & - & - & - \\
\hline$P S V$ & + & + & - & - & - & - & + & + & - & - & - \\
\hline$P V$ & - & + & + & + & + & + & - & - & - & - & - \\
\hline$H O$ & - & + & + & - & - & - & + & + & + & + & + \\
\hline$A T P V$ & - & + & - & - & - & - & - & - & - & - & - \\
\hline
\end{tabular}

Table 1: A * means that the property is satisfied and used to characterize the corresponding value. The + sign means that the property is satisfied, but not used in the characterization, and the - sign means that it is not satisfied.

A few comments are in order. First, beyond EFF, ADD, and NSP, all the properties introduced in this paper are satisfied by at least one of the five solutions taken from the literature. Second, among the latter, $H O$ seems to be the solution that has more features in common with the solutions based on levels structures, as it satisfies all lower bound properties, IMT, SNSP, and NOP. Third, except for EFF, ADD, and NSP, ATPV does not satisfy any of the other properties considered in the paper.

Finally, to further illustrate the differences between our three new solutions and the five solutions from the literature, we compute the payoffs for the latter in the case of the game with hierarchical structure of Example 3.1. There are two polar cases: either the spoils are divided

\footnotetext{
${ }^{19}$ For a complete definition of the Average Tree Permission Value, we refer to van den Brink et al. (2015).
} 
equally or everything is allocated to the root of the tree. Indeed,

$$
\begin{aligned}
& \operatorname{Sh}\left(N, u_{N}\right)=\operatorname{PV}\left(N, u_{N}, D\right)=\operatorname{ATPV}\left(N, u_{N}, D\right)=\left(\frac{1}{8}, \frac{1}{8}, \frac{1}{8}, \frac{1}{8}, \frac{1}{8}, \frac{1}{8}, \frac{1}{8}, \frac{1}{8}\right), \\
& \operatorname{PSV}\left(N, u_{N}, D\right)=\operatorname{HO}\left(N, u_{N}, D\right)=(1,0,0,0,0,0,0,0) .
\end{aligned}
$$

That is, at least as far as unanimity games are concerned, our three solutions use the entire information contained in the hierarchical structure $(S h, P V$ and $A T P V$ do not differentiate at all between the players), but not so much as to assign all the worth to one player (as done by $P S V$ and HO). Accordingly, there is a sense in which $\varphi^{S L}, \bar{\varphi}^{S L}$, and $\widetilde{\varphi}^{S L}$ yield a compromise between the two extreme proposals. Such a feature is a consequence of the fact that our solutions are based on the Shapley Levels Value. As such, they are more sensitive to the agents' position in the hierarchy.

The importance of how responsive solutions should be to changes in the hierarchy seems to have been underestimated in the literature on games with hierarchical structure. When the game is the unanimity game of the grand coalition, in particular, the payoff assigned by a solution to a player in a game with hierarchical structure can be interpreted as the player's importance in the hierarchy. Our paper adds to the knowledge about this issue by providing three solution concepts that assign a distinctive payoff to each player, depending not only on her position in the hierarchy but also on that of certain other players. Hence, our approach builds on the assumption that even small changes in the hierarchy could induce changes in the payoffs.

\section{Concluding Remarks}

In this paper we have introduced a new way to exploit the information contained in a hierarchical structure by mapping it into a levels structure, without any loss of information in many settings. This has enabled us to define three new solutions for games with hierarchical structure, which we have all characterized. From an axiomatic point of view, the most relevant differences between the solutions stem from the fulfillment (or lack of fulfillment) of certain properties that result from relaxing, by means of the information contained in the hierarchical structure, the requirement that in a monotone game, a necessary player should obtain at least as much as any other player.

Because the latter requirement (i.e., the Necessary Player Property) and the Null Player Property combined are too demanding in our setting - when taken together with Efficiency and 
Additivity - , we have chosen to keep the Null Player Property and to relax the Necessary Player Property. We have then shown that doing so fits particularly well with the approach based on levels structures, for it has allowed us to define reasonable solutions for games with hierarchical structure. Instead, we could have kept the Necessary Player Property and considered different ways to weaken the Null Player Property. This is a plan for future research.

One further appeal for the suggested ways of weakening the Necessary Player Property is that they are all informative about how certain relations between players and/or teams translate into power within hierarchical organizations. According to our assessment, these relations have been overlooked to date. Gaining insight about whether, and if so how, an organization's structure should affect the rewards to its members might be a relevant issue in real-world organizations such as universities, firms, or countries. Our paper contributes to this knowledge by shedding more light on the comparison of different organizational structures, some of which are flat and some of which are structured along steep hierarchies.

Our analysis has relied in particular on three ways of mapping a directed tree into a levels structure. In all cases, we have required that a player who is higher in the hierarchy is more independent from the other players in the collection of nested partitions, in the sense that such a player becomes a singleton coalition at a lower-ranked partition than a player that is lower in the hierarchy. A full understanding of the advantages of our procedure requires further examination. For instance, it seems interesting to answer a question that can be seen as the reversal of this paper: Which are all the solutions for games with hierarchical structure that can be obtained according to our procedure? This is also left for future research.

\section{Acknowledgements}

Mikel Álvarez-Mozos and Oriol Tejada acknowledge the financial support of Ministerio de Economía y Competitividad through projects MTM2014-53395-C3-2-P and ECO2014-52340-P as well as from Generalitat de Catalunya through project 2014-SGR-40. Discussions with Hans Gersbach and Eyal Winter are acknowledged. All errors are our own. 


\section{References}

Álvarez-Mozos, M., van den Brink, R., van der Laan, G., and Tejada, O. (2015). From Hierarchies to Levels: New solutions for Games with Hierarchical Structure. CER-ETH-Center of Economic Research at ETH Zurich, Working Paper, 15:215.

Álvarez-Mozos, M. and Tejada, O. (2011). Parallel Characterizations of a Generalized Shapley Value and a Generalized Banzhaf Value for Cooperative Games with Levels Structure of Cooperation. Decision Support Systems, 52:21-27.

Álvarez-Mozos, M., van den Brink, R., van der Laan, G., and Tejada, O. (2013). Share Functions for Cooperative Games with Levels Structure of Cooperation. European Journal of Operational Research, 224:167-179.

Ambec, S. and Sprumont, Y. (2002). Sharing a River. Journal of Economic Theory, 107:453-462.

Aumann, R. J. and Drèze, J. (1974). Cooperative Games with Coalition Structures. International Journal of Game Theory, 3:217-237.

Curiel, I., Pederzoli, G., and Tijs, S. (1989). Sequencing Games. European Journal of Operational Research, 40:344-351.

Demange, G. (2004). On Group Stability in Hierarchies and Networks. Journal of Political Economy, 112:754-778.

Faigle, U. and Kern, W. (1992). The Shapley Value for cooperative games under precedence constraints. International Journal of Game Theory, 21(3):249-266.

Fiestras-Janeiro, M., Sánchez-Rodríguez, E., and Schuster, M. (2016). A precedence constraint value revisited. TOP, 24:156-179.

Gilles, R. and Owen, G. (1994). Cooperative Games and Disjunctive Permission Structures. Technical report, Virginia Polytechnic Institute and State University.

Gilles, R., Owen, G., and van den Brink, R. (1992). Games with Permission Structures: The Conjunctive Approach. International Journal of Game Theory, 20:277-293. 
Graham, D., Marshall, R., and Richard, J. (1990). Differential Payments within a Bidder Coalition and the Shapley Value. American Economic Review, 80:493-510.

Harsanyi, J. (1959). A Bargaining Model for Cooperative $n$-Person Games. In Tucker, A. and Luce, R., editors, Contributions to the Theory of Games II. Princeton University Press.

Hart, S. and Kurz, M. (1983). Endogeneous Formation of Coalitions. Econometrica, 51:10471064.

Herings, P. J.-J., Van Der Laan, G., and Talman, D. (2005). The positional power of nodes in digraphs. Social Choice and Welfare, 24(3):439-454.

Littlechild, S. and Owen, G. (1973). A Simple Expression for the Shapley Value in a Special Case. Management Science, 20:370-372.

Maniquet, F. (2003). A Characterization of the Shapley Value in Queueing Problems. Journal of Economic Theory, 109:90-103.

Mishra, D. and Talman, A. (2010). A characterization of the average tree solution for tree games. International Journal of Game Theory, 39:105-111.

Ni, D. and Wang, Y. (2007). Sharing a Polluted River. Games and Economic Behavior, 60:176186.

Owen, G. (1977). Values of Games with a Priori Unions. In Henn, R. and Moeschlin, O., editors, Mathematical Economics and Game Theory, pages 76-88. Springer-Verlag.

Shapley, L. S. (1953). A Value for n-Person Games. In Tucker, A. W., editor, Contributions to the Theory of Games II, pages 307-317. Princeton University Press.

van den Brink, R. (1997). An Axiomatization of the Disjunctive Permission Value for Games with a Permission Structure. International Journal of Game Theory, 26:27-43.

van den Brink, R. (2010). Axiomatizations of Banzhaf permission values for games with a permission structure. International Journal of Game Theory, 39:445-466.

van den Brink, R., Dietz, C., van der Laan, G., and Xu, G. (2016). Comparable characterizations of four solutions for permission tree games. Economic Theory. 
van den Brink, R. and Gilles, R. (1996). Axiomatization of the Conjunctive Permission Value for Games with Permission Structures. Games and Economic Behavior, 12:113-126.

van den Brink, R., Herings, P., van der Laan, G., and Talman, A. (2015). The Average Tree Permission Value for Games with a Permission Structure. Economic Theory, 58:99-123.

Williamson, O. E. (1967). Hierarchical control and optimum firm size. Journal of Political Economy, 75:123-138.

Winter, E. (1989). A Value for Cooperative Games with Levels Structure of Cooperation. International Journal of Game Theory, 18:227-240. 


\section{Appendix A Proofs of Section 3}

Proof of Theorem 3.2 We note that $|N| \geq 2$. We first prove that for each $(N, D) \in \mathcal{D}$, every partition $P \in B^{D}$ satisfies P1-P5. Second, we prove that if a partition $P \neq\{N\}$ respects a given directed tree $(N, D) \in \mathcal{D}$ (i.e., the partition satisfies $\mathrm{P} 1-\mathrm{P} 5$ with respect to $(N, D))$, then $P$ must belong to $B^{D}$.

Existence: Let $(N, D) \in \mathcal{D}, i \in N$ and $P$ be any proper partition of the levels structure $\mathcal{H}(N, D)$. We assume that $P \neq\{\{j\}\}_{j \in N}$, for if not it is immediate to verify that $P$ satisfies all properties with respect to any $D$. We distinguish two cases, depending on whether $P=B_{s, 1}^{D}$ as defined in Eq. (3.3) or $P=B_{s, 2}^{D}$ as defined in Eq. (3.4), where $s \geq 1$ is an integer.

Case 1: $T=B_{s, 1}^{D}$. In this case, we have

$$
P=\{\{i\}: l(i) \leq s-1\} \cup\left\{\widehat{S}_{D}(i): l(i)=s-1\right\}
$$

First, let $i, j \in N$ be such that $(i, j) \in D$ and $P_{i} \neq\{i\} .{ }^{20}$ Then, by Eq. (A.5), we obtain $l(i) \geq s$ and then $\widehat{S}_{D}(i) \subseteq P_{i}$. In particular, P1 is satisfied. Second, let $i, j \in N$ be such that $j \in A(i)$ and $P_{i} \backslash \widehat{S}_{D}(i) \neq \emptyset$. By Eq. (A.5), we have $l(i) \geq s$. Since $l(j)=l(i)$, from Eq. (A.5) it follows that $P_{i}=P_{j}$ and P2 holds. Third, let $i, j \in N$ be such that $l(i)=l(j), S_{D}(i) \neq \emptyset$, and $P_{i}=\{i\}$. From Eq. (A.5), it follows that $l(i) \leq s-1$, so $P_{j}=\{j\}$ and P3 is met. Fourth, let $i, j \in N$ be such that $(i, j) \in D$ and $P_{i}=\{i\}$. Note in particular that $S_{D}(i) \neq \emptyset$. Then, $l(i) \leq s-1$ and $l(j) \leq s$. If $l(i)<s-1$, we have $P_{j}=\{j\}$. If $l(i)=s-1$, we have $P_{j}=\widehat{S}_{D}(i)$. In any case, P4 is satisfied. Fifth, let $i, j \in N$ be such that $l(i)=l(j), A(i) \backslash i \neq \emptyset$, and $A(j) \backslash j \neq \emptyset$. If $l(i)<s$, we have $P_{i}=\{i\}$ and $P_{j}=\{j\}$. If $l(i) \geq s$, we have $A(i) \subseteq P_{i}$ and $A(j) \subseteq P_{j}$. In the two cases, P5 is satisfied.

Case 2: $P=B_{s, 2}^{D}$. In this case, we have

$$
P=\{\{i\}: l(i) \leq s-1\} \cup\left\{\widehat{S}_{D}(j) \cup j: l(j)=s\right\}
$$

First, let $i, j \in N$ be such that $(i, j) \in D$ and $P_{i} \neq\{i\}$. Then, by Eq. (A.6), we have $l(i) \geq s$ and $P_{i}=P_{j}$, so $\mathrm{P} 1$ is satisfied. Second, let $i, j \in N$ be such that $j \in A(i)$ and $P_{i} \backslash\left(\widehat{S}_{D}(i) \cup i\right) \neq \emptyset$. By Eq. (A.6), we have $l(i) \geq s+1$. Then, $P_{i}=P_{j}$ and P2 holds. Third, let $i, j \in N$ be such that $l(i)=l(j)$, $S_{D}(i) \neq \emptyset$, and $P_{i}=\{i\}$. Then, Eq. (A.6) implies that $l(j)=l(i) \leq s-1$ and hence $P_{j}=\{j\}$. Therefore, P3 is met. Fourth, let $i, j \in N$ be such that $(i, j) \in D$ and $P_{i}=\{i\}$. Note in particular that

\footnotetext{
${ }^{20}$ Recall that for a given partition of $N, P$ and $i \in N$, we denote by $P_{i}$ the element of $P$ containing player $i$.
} 
$S_{D}(i) \neq \emptyset$ and then, $l(i) \leq s-1$. If $l(i)=s-1$, we have $P_{j}=\widehat{S}_{D}(j) \cup j \subseteq \widehat{S}_{D}(i)$. If $l(i)<s-1$, we have $l(j) \leq s-1$ and hence $P_{j}=\{j\} \subseteq \widehat{S}_{D}(i)$. In any case, P4 is satisfied. Fifth, let $i, j \in N$, with $i \neq j$, be such that $l(i)=l(j), A(i) \backslash i \neq \emptyset$, and $A(j) \backslash j \neq \emptyset$. If $l(i) \leq s-1$, we obtain $P_{i}=\{i\}$ and $P_{j}=\{j\}$. If $l(i)=s$, we obtain $P_{i}=\widehat{S}_{D}(i) \cup i$ and $P_{j}=\widehat{S}_{D}(j) \cup j$. If $l(i)>s$, we obtain $P_{i} \nsubseteq \widehat{S}_{D}(i)$ and $P_{j} \nsubseteq \widehat{S}_{D}(j)$. In all three cases, P5 holds.

Uniqueness: Let $P$ be a proper partition of $N$ that satisfies $\mathrm{P} 1-\mathrm{P} 5$. First, let $i \in N$ be such that $P_{i} \neq\{i\}$. By $\mathrm{P} 1, P_{i}=P_{j}$ for every $j \in S_{D}(i)$. Thus, also $P_{j} \neq\{j\}$, and applying $\mathrm{P} 1$ again yields $P_{k}=P_{j}$ for every $k \in S_{D}(j)$. By repeating the same procedure until there is no more agent with successors, we obtain that $P_{l}=P_{i}$ for every $l \in \widehat{S}_{D}(i)$. Hence the next implication holds for every $i \in N$ :

$$
\left[P_{i} \neq\{i\}\right] \Longrightarrow\left[\widehat{S}_{D}(i) \subseteq P_{i}\right]
$$

Next, for given $i$ and $j \in \widehat{P}_{D}(i)$, suppose that $P_{j} \neq\{j\}$. Then by Eq. (A.7) it holds that $i \in P_{j}$, and thus $P_{i} \neq\{i\}$. From this, we obtain the implication below for every $i \in N$

$$
\left[P_{i}=\{i\}\right] \Longrightarrow\left[\forall j \in \widehat{P}_{D}(i), P_{j}=\{j\}\right]
$$

We can assume that there is at least one player who is a singleton in $P$. Conversely, suppose that for every $l \in N, P_{l} \neq\{l\}$. In particular, $P_{i_{0}} \neq\left\{i_{0}\right\}$. Since $\widehat{S}_{D}\left(i_{0}\right)=N \backslash\left\{i_{0}\right\}$, from Eq. (A.7) it follows that $P=\{N\}$, and thus $P$ is not a proper partition of $N$.

Define the set $\mathcal{K}^{D}(P) \subseteq N$ by

$$
\mathcal{K}^{D}(P)=\left\{i \in N: P_{i}=\{i\}, S_{D}(i) \neq \emptyset, \text { and } \exists j \in S_{D}(i) \text { such that } P_{j} \neq\{j\}\right\}
$$

We show that either $\mathcal{K}^{D}(P) \neq \emptyset$ or

$$
P=\{\{i\}: i \in N\}
$$

In the latter case, $P=B_{l(D), 2}^{D}$ and thus $P \in B^{D}$, which concludes the proof.

If $\mathcal{K}^{D}(P)=\emptyset$, suppose there is a player $i$ with $P_{i}=\{i\}$ and $S_{D}(i) \neq \emptyset$. Then P3 implies that $P_{j}=\{j\}$ for every $j \in N$, where $l(j)=l(i)$. By Eq. (A.8), it then follows that $P_{k}=\{k\}$ for every $k \in N$ where $l(k)<l(i)$. Further, for every $j \in N$ such that $l(j)=l(i)$ and $S_{D}(j) \neq \emptyset$, we have that $P_{k}=\{k\}$ for every $k \in S_{D}(j)$, since otherwise $j \in \mathcal{K}^{D}(P)$, which contradicts that $\mathcal{K}^{D}(P)=\emptyset$. Hence, $P_{k}=\{k\}$ for every $k \in N$ with $l(k)=l(i)+1$. By repeating the latter step iteratively, it follows that Eq. (A.10) holds. It remains to consider the case where $S_{D}(i)=\emptyset$ for all $i \in N$, with $P_{i}=\{i\}$. Since there is at least one player $i$ with $P_{i}=\{i\}$, from Eq. (A.8) it follows that $P_{i_{0}}=\left\{i_{0}\right\}$. As $|N| \geq 2$ and 
$\mathcal{K}^{D}(P)=\emptyset$, we obtain again following an iterative argument that Eq. (A.10) holds, hence completing the proof.

In the remaining, we consider the case where $\mathcal{K}^{D}(P)$ is nonempty. We first prove that

$$
l(i)=l(j) \text { for } i, j \in \mathcal{K}^{D}(P),
$$

i.e., all players of $\mathcal{K}^{D}(P)$ are at the same distance from the root. Suppose this is not the case, and let $i, j \in \mathcal{K}^{D}(P)$ be such that $l(i)<l(j)$. Let $k \in S_{D}(i)$ be a successor of $i$ such that $P_{k} \neq\{k\}$. The existence of such a player is guaranteed, as $i \in \mathcal{K}^{D}(P)$. When $l(i)+1=l(j)$, we obtain $l(k)=l(j)$. Since $j \in \mathcal{K}^{D}(P)$, and so $S_{D}(j) \neq \emptyset$ and $P_{j}=\{j\}$, P3 implies that $P_{k}=\{k\}$, which is a contradiction. When $l(i)+1<l(j)$, let $h \in \widehat{P}_{D}(j)$ be such that $l(h)=l(i)+1$. Since $j \in \mathcal{K}_{D}^{T}, P_{h}=\{h\}$ by Eq. (A.8). By P3, also $P_{k}=\{k\}$, again contradicting the hypothesis above. Therefore, Eq. (A.11) holds.

Next, we consider the set of all players who are subordinates of some player in $\mathcal{K}^{D}(P)$, i.e.,

$$
S\left(\mathcal{K}^{D}(P)\right)=\left\{i \in N: \exists j \in \mathcal{K}^{D}(P) \text { such that } i \in \widehat{S}_{D}(j)\right\}
$$

We deal with the players in this set and the players in her complement separately.

First, we consider the set $N \backslash S\left(\mathcal{K}^{D}(P)\right)$. Since $\mathcal{K}^{D}(P) \neq \emptyset$, it follows by P3 that $P_{i}=\{i\}$ for every $i \in N \backslash \mathcal{K}^{D}(P)$ with $l(i)=s$, and consequently, $P_{i}=\{i\}$ for every $i \in N$ with $l(i)=s$. Further, by Eq. (A.8), we obtain that $P_{i}=\{i\}$ for every $i \in N$ with $l(i)<s$. Next, consider a player $i \in N \backslash \mathcal{K}^{D}(P)$ such that $l(i)=s$ and $S_{D}(i) \neq \emptyset$ and take $j \in S_{D}(i)$. Since, $i \notin \mathcal{K}^{D}(P)$, it holds that $P_{j}=\{j\}$. By repeating the latter argument for every $k \in S_{D}(j)$ and so forth, it follows that $P_{k}=\{k\}$ for all $k \in \widehat{S}_{D}(i)$. Taking everything together, we have shown that for every $j \in N \backslash S\left(\mathcal{K}^{D}(P)\right)$,

$$
P_{j}=\{j\} .
$$

Second, we consider the set $S\left(\mathcal{K}^{D}(P)\right)$. Take some $i \in \mathcal{K}^{D}(P)$ and suppose that for some $j \in S_{D}(i)$, it holds that $P_{j} \backslash\left(\widehat{S}_{D}(j) \cup j\right) \neq \emptyset$, and thus $P_{j} \neq\{j\}$. By Eq. (A.7), it holds that $\widehat{S}_{D}(j) \subseteq P_{j}$. Then, since $P_{i} \neq\{i\}$, by $\mathrm{P} 4$, we have

$$
P_{j} \subseteq \widehat{S}_{D}(i)
$$

On the other hand, when $A(j)=\{j\}$, it holds trivially that

$$
\bigcup_{k \in S_{D}(i)}\left(\widehat{S}_{D}(k) \cup k\right)=\widehat{S}_{D}(i) \subseteq P_{j}
$$

and thus $P_{j}=\widehat{S}_{D}(i)$. Also, if $A(j) \backslash j \neq \emptyset$, then, by P2, $k \in P_{j}$ for all $k \in A(j) \backslash j$ and Eq. (A.7) implies that $\widehat{S}_{D}(l) \subseteq P_{j}$ for all $k \in A(j) \backslash j$. Therefore,

$$
\bigcup_{k \in S_{D}(i)}\left(\widehat{S}_{D}(k) \cup k\right)=\widehat{S}_{D}(i) \subseteq P_{j}
$$


and then, $P_{j}=\widehat{S}_{D}(i)$. Hence, when there exists $j \in S_{D}(i)$ such that $P_{j} \backslash\left(\widehat{S}_{D}(j) \cup j\right) \neq \emptyset$, it holds that $P_{j}$ includes all subordinates of $i$, and thus for every $k \in S_{D}(i)$,

$$
P_{k}=\widehat{S}_{D}(i)
$$

Next, suppose that for some $i \in \mathcal{K}^{D}(P)$, it holds that $P_{j} \subseteq \widehat{S}_{D}(j)$ for every $j \in S_{D}(i)$. If $P_{j} \neq\{j\}$, then Eq. (A.7) implies that $\widehat{S}_{D}(j) \subseteq P_{j}$ and thus $P_{j}=\widehat{S}_{D}(j)$. When $P_{j}=\{j\}$ and $S_{D}(j) \neq \emptyset$, we obtain by P3 that $P_{k}=\{k\}$ for every $k \in S_{D}(i)$, which contradicts that $i \in \mathcal{K}^{D}(P)$. Hence, when $P_{j}=\{j\}, S_{D}(j)=\emptyset$. Therefore, $P_{j}=\widehat{S}_{D}(j) \cup j$. In both cases, we have that $P_{j}=\widehat{S}_{D}(j) \cup j$. It follows that for every $i \in \mathcal{K}^{D}(P)$, either Eq. (A.13) holds, or for all $j \in S_{D}(i)$,

$$
P_{j}=\widehat{S}_{D}(j) \cup j
$$

Finally, let $i_{1}, i_{2} \in \mathcal{K}^{D}(P)$ be two different players and suppose that Eq. (A.14) holds when we take $i=i_{1}$, but it does not hold when we take $i=i_{2}$, that Eq. (A.13) holds when we take $i=i_{2}$, but that it does not hold when we take $i=i_{1}$. It follows, in particular, that $A\left(i_{1}\right) \backslash\left\{i_{1}\right\} \neq \emptyset$ and $A\left(i_{2}\right) \backslash\left\{i_{2}\right\} \neq \emptyset$. However, this leads to a contradiction with P5.

As a consequence of all the above steps, we have proved that either

$$
P=\bigcup_{i \in N: l(i)=s}\left[\{\{i\}\} \cup\left(\bigcup_{j \in \widehat{P}_{D}(i)}\{\{j\}\}\right) \cup\left\{\widehat{S}_{D}(i)\right\}\right]
$$

or

$$
P=\bigcup_{i \in N: l(i)=s}\left[\{\{i\}\} \cup\left(\bigcup_{j \in \widehat{P}_{D}(i)}\{\{j\}\}\right) \cup\left(\bigcup_{j \in S_{D}(i)}\left\{\widehat{S}_{D}(j) \cup\{j\}\right\}\right)\right] .
$$

Note that Eqs. (A.15) and (A.16) are equivalent to $P=B_{1, s}^{D}$ and $P=B_{2, s}^{D}$ respectively, so that $P \in B^{D}$.

\section{Independence of the properties used in Theorem 3.2}

Consider the following triples composed of a finite set $N$, a rooted tree $D \in \mathcal{G}^{N}$, and a partition $T \in \mathcal{P}^{N}:$

1. $N=\{1,2,3\}, D=\{(1,2),(2,3)\}$ and $T=\{\{1,2\},\{3\}\}$.

In this case, $D$ and $T$ satisfy $\mathrm{P} 2, \mathrm{P} 3, \mathrm{P} 4$, and $\mathrm{P} 5$ but fail to satisfy $\mathrm{P} 1$.

2. $N=\{1,2,3,4,5\}, D=\{(1,2),(1,3),(1,4),(1,5)\}$ and $T=\{\{1\},\{2,3\},\{4,5\}\}$.

In this case, $D$ and $T$ satisfy $\mathrm{P} 1, \mathrm{P} 3, \mathrm{P} 4$, and $\mathrm{P} 5$ but fail to satisfy $\mathrm{P} 2$. 
3. Case 3: $N=\{1,2,3,4,5\}, D=\{(1,2),(1,3),(2,4),(3,5)\}$ and $T=\{\{1\},\{2,4\},\{3\},\{5\}\}$.

In this case, $D$ and $T$ satisfy $\mathrm{P} 1, \mathrm{P} 2, \mathrm{P} 4$, and $\mathrm{P} 5$ but fail to satisfy $\mathrm{P} 3$.

4. $N=\{1,2,3,4,5\}, D=\{(1,2),(1,3),(2,4),(3,5)\}$ and $T=\{\{1\},\{2\},\{3\},\{4,5\}\}$.

In this case, $D$ and $T$ satisfy P1, P2, P3, and P5 but fail to satisfy P4.

5. $N=\{1,2,3,4,5,6,7\}, D=\{(1,2),(2,4),(2,5),(1,3),(3,6),(3,7)\}$ and $T=\{\{1\},\{2\},\{3\},\{4,5\},\{6\},\{7\}\}$.

In this case, $D$ and $T$ satisfy $\mathrm{P} 1, \mathrm{P} 2, \mathrm{P} 3$, and $\mathrm{P} 4$ but fail to satisfy $\mathrm{P} 5$. 


\section{Appendix B Proofs of Section 4}

This appendix contains the proof of Theorem I. Before we present it, however, we prove two lemmas that will come in handy in the proofs of Theorems I-III. Given $(N, D) \in \mathcal{D}$ and $i \in N$, let $N_{i}$ be given by

$$
N_{i}=\widehat{S}_{D}(i) \cup \widehat{P}_{D}(i) \cup S_{D}\left(\widehat{P}_{D}(i)\right),
$$

Note that $i \in S_{D}\left(\widehat{P}_{D}(i)\right)$ and thus $i \in N_{i}$, and that $N_{i}$ further consists of all subordinates and superiors of $i$ together with all successors of her superiors. We call the players in $N_{i} \backslash i$ the relatives of $i{ }^{21}$ We next define the game $\left(N_{i}, v_{i}\right) \in \mathcal{G}$ by, $v_{i}(T)=v\left(T_{i}^{D}\right)$ for every $T \subseteq N_{i}$, with

$$
T_{i}^{D}=T \cup\left(\bigcup_{j \in T \backslash\left(\widehat{S}_{D}(i) \cup \widehat{P}_{D}(i) \cup i\right)} \widehat{S}_{D}(j)\right) .
$$

We prove now the first of our lemmas.

Lemma B.1. If a solution on $\mathcal{G D}, f$, satisfies independence of merging teams (IMT), then for every $(N, v, D) \in \mathcal{G D}$ and $i \in N, f_{i}(N, v, D)=f_{i}\left(N_{i}, v_{i}, D_{N_{i}}\right)$.

\section{Proof.}

Let $(N, v, D) \in \mathcal{G D}$ and $i \in N$, and consider $\left(N_{i}, v_{i}, D_{N_{i}}\right)$ as defined in Eqs. (B.17) and (B.18). If $N_{i}=N$, then $D_{N_{i}}=D$ and $T_{i}^{D}=T$ for all $T$, so that $v_{i}=v$. Hence $f_{i}\left(N_{i}, v_{i}, D_{N_{i}}\right)=f_{i}(N, v, D)$. Hence, consider the case in which $N_{i} \neq N$. Then, for some integer $m>0$, there is a sequence of games $\left(N^{(k)}, v^{(k)}, D^{(k)}\right), k=0, \ldots, m$, such that (i) $\left(N^{(0)}, v^{(0)}, D^{(0)}\right)=(N, v, D)$, (ii) for $k=1, \ldots m$, $N^{(k)}=N^{(k-1)} \backslash \widehat{S}_{D}(j)$ for some $j \in N^{(k-1)}, v^{(k)}=\left(v^{(k-1)}\right)^{k}$ and $D^{(k)}=\left(D^{(k-1)}\right)_{N^{(k)}}$, and (iii) $N^{(m)}=N_{i}$ and so $D^{(m)}=D_{N_{i}}$. The existence of such a sequence is guaranteed by Eq. (B.17). Then, by IMT, for all $k \in\{1, \ldots, m\}$,

$$
f_{i}\left(N^{(k)}, v^{(k)}, D^{(k)}\right)=f_{i}\left(N^{(k-1)}, v^{(k-1)}, D^{(k-1)}\right) \text { for all } i \in N^{(m)}=N_{i} .
$$

Moreover, for each $T \subseteq N_{i}$,

$$
v^{(m)}(S)=v^{(0)}\left(\bigcup_{j \in T \backslash\left(\widehat{S}_{D}(i) \cup \widehat{P}_{D}(i) \cup i\right)} \widehat{S}_{D}(j)\right) .
$$

Since $v^{(0)}=v$, it follows that $v^{(m)}=v_{i}$, which completes the proof.

\footnotetext{
${ }^{21}$ Note that "being a relative" is not a symmetric relation.
} 
When all players in $N \backslash i$ are relatives of $i$, we have $N_{i}=N$ and thus $\left(N_{i}, v_{i}, D_{N_{i}}\right)=(N, v, D)$. In that case, the statement of the lemma is trivial. When they are not relatives, the lemma provides us with a property implied by IMT requiring the payoff of a player not be affected by changes in the position in the hierarchy of players who are not her relatives. As the proof of Lemma B.1 shows, $\left(N_{i}, v_{i}, D_{N_{i}}\right)$ is obtained from the repeated application of IMT. Indeed, when $N_{i} \neq N$, there is an integer $m>0$ and a sequence of games with hierarchical structure $\left(N^{(k)}, v^{(k)}, D^{(k)}\right)$, with $k=0, \ldots, m$, such that (i) $\left(N^{(0)}, v^{(0)}, D^{(0)}\right)=(N, v, D)$, (ii) for every $k \in\{1, \ldots m\}, N^{(k)}=N^{(k-1)} \backslash \widehat{S}_{D}(j)$ for some $j \in N^{(k-1)}$, $v^{(k)}=\left(v^{(k-1)}\right)^{j}$, and $D^{(k)}=\left(D^{(k-1)}\right)_{N^{(k)}}$, and (iii) $\left(N^{(m)}, v^{(m)}, D^{(m)}\right)=\left(N_{i}, v_{i}, D_{N_{i}}\right)$.

The next example illustrates the consequences of IMT as stated in Lemma B.1.

Example B.2. Consider $\left(N, u_{N}, D\right) \in \mathcal{G D}$ with $D$ as given in Example 3.1. For $i=4$, we have $N_{4}=\{1,2,3,4,5\}$ and $\left(N_{4}, D_{N_{4}}\right)$ the tree given by the left side of Figure 2. If a solution $f$ satisfies IMT, then Lemma B.1 implies that the payoff to Player 4 in $(N, v, D)$ is equal to the payoff of Player 4 in $\left(N_{4}, v_{4}, D_{N_{4}}\right)$. Hence, $\varphi_{4}^{S L}\left(N_{4},\left(u_{N}\right)_{4}, D_{N_{4}}\right)=\varphi_{4}^{S L}\left(N, u_{N}, D\right)=\frac{1}{16}$. Taking $i=3$ yields $N_{3}=$ $\{1,2,3,6\}$ and $\left(N_{3}, D_{N_{3}}\right)$, given by the right side of Figure 2. By Lemma B.1, $\varphi_{3}^{S L}\left(N_{3},\left(u_{N}\right)_{3}, D_{N_{3}}\right)=$ $\varphi_{3}^{S L}\left(N, u_{N}, D\right)=\frac{1}{8}$.
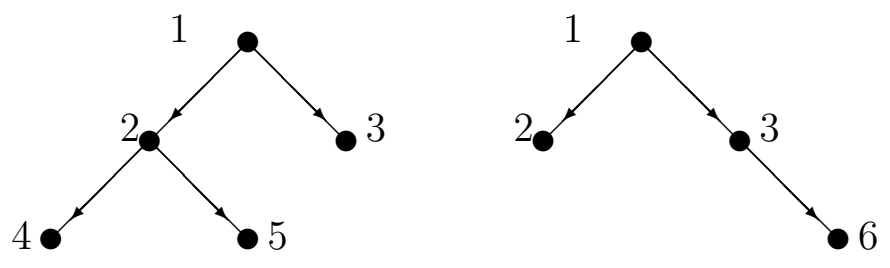

Figure 2: Directed trees $D_{N_{4}}$ and $D_{N_{3}}$ of Example 3.1

The second of our lemmas shows that for a unanimity game $\left(N, u_{T}\right)$, the repeated application of IMT results in a new unanimity game. Note that $T(i)=T$ when $N_{i}=N$.

Lemma B.3. Consider $\left(N, c u_{T}, D\right) \in \mathcal{G D}$, where $c>0$ and $T \subseteq N$. Then, for every $i \in N$, $\left(N_{i},\left(c u_{T}\right)_{i}\right)=\left(N_{i}, c u_{T(i)}\right)$ with

$$
T(i)=\left(T \cap N_{i}\right) \cup\left\{j \in N_{i} \backslash\left(\widehat{S}_{D}(i) \cup \widehat{P}_{D}(i) \cup i\right): \widehat{S}_{D}(j) \cap T \neq \emptyset\right\} .
$$




\section{Proof.}

Let $\left(N, c u_{T}, D\right) \in \mathcal{G D}$, with $c>0$ and $T \subseteq N$, and let $i \in N$. For each $R \subseteq N_{i}$, we have

$$
\left(u_{T}\right)_{i}(R)=u_{T}\left(R_{i}^{D}\right)= \begin{cases}1 & \text { if } T \subseteq R_{i}^{D} \\ 0 & \text { otherwise }\end{cases}
$$

where $R_{i}^{D}$ is defined according to Eq. (B.18). Note that

$$
\begin{aligned}
T \subseteq R_{i}^{D} & \Longleftrightarrow T \subseteq R \cup\left(\bigcup_{j \in R \backslash\left(\widehat{S}_{D}(i) \cup \widehat{P}_{D}(i) \cup i\right)} \widehat{S}_{D}(j)\right) \\
& \Longleftrightarrow\left[T \cap N_{i} \subseteq R\right] \text { and }\left[T \backslash N_{i} \subseteq \underset{j \in R \backslash\left(\widehat{S}_{D}(i) \cup \widehat{P}_{D}(i) \cup i\right)}{\bigcup_{D}(j)}\right]
\end{aligned}
$$

We claim that

$$
\begin{aligned}
& {\left[T \backslash N_{i} \subseteq \underset{j \in R \backslash\left(\widehat{S}_{D}(i) \cup \widehat{P}_{D}(i) \cup i\right)}{\bigcup} \widehat{S}_{D}(j)\right] } \\
\Longleftrightarrow & {\left[\left\{j \in N_{i} \backslash\left(\widehat{S}_{D}(i) \cup \widehat{P}_{D}(i) \cup i\right): \widehat{S}_{D}(j) \cap T \neq \emptyset\right\} \subseteq R\right] . }
\end{aligned}
$$

Then, $\left(c u_{T}\right)_{i}=c u_{T(i)}$ with

$$
T(i)=\left(T \cap N_{i}\right) \cup\left\{j \in N_{i} \backslash\left(\widehat{S}_{D}(i) \cup \widehat{P}_{D}(i) \cup i\right): \widehat{S}_{D}(j) \cap T \neq \emptyset\right\} .
$$

Therefore, it only remains to prove the claim in Eq. (B.19). On the one hand, let $j \in N_{i} \backslash\left(\widehat{S}_{D}(i) \cup \widehat{P}_{D}(i) \cup i\right)$ be such that $\widehat{S}_{D}(j) \cap T \neq \emptyset$ and $j \notin R$. Take $k \in \widehat{S}_{D}(j) \cap T$. Then, by construction of $N_{i}$-note that $j \in N_{i} \backslash\left(\widehat{S}_{D}(i) \cup \widehat{P}_{D}(i) \cup i\right)$ - we have $k \in T$ and $k \notin N_{i}$, but

$$
k \notin \bigcup_{h \in R \backslash\left(\widehat{S}_{D}(i) \cup \widehat{P}_{D}(i) \cup i\right)} \widehat{S}_{D}(h) .
$$

On the other hand, let $k \in T \backslash N_{i}$ such that Eq. (B.20) holds. Then, let $j \in N_{i} \backslash\left(\widehat{S}_{D}(i) \cup \widehat{P}_{D}(i) \cup i\right)$ be such that $k \in \widehat{S}_{D}(j)$. The existence of $j$ is guaranteed by construction of $N_{i}$. Hence,

$$
j \in\left\{h \in N_{i} \backslash\left(\widehat{S}_{D}(i) \cup \widehat{P}_{D}(i) \cup i\right): \widehat{S}_{D}(h) \cap T \neq \emptyset\right\} \backslash R .
$$

We are finally in the position to prove the characterization of the first solution, $\varphi^{S L}$.

\section{Proof of Theorem I}

We first prove that there is at most one solution on $\mathcal{G D}$ that satisfies all properties. After that, we will prove that $\varphi^{S L}$ satisfies them. 
Uniqueness: Suppose that $f$ satisfies the seven axioms. For every $\left(N, v_{0}, D\right)$, with $\left(N, v_{0}\right)$ the null game given by $v_{0}(S)=0$ for all $S \subseteq N$, NPP implies that $f_{i}\left(N, v_{0}, D\right)=0$ for all $i \in N$.

Next, let $\left(N, c u_{T}, D\right) \in \mathcal{G D}$, where $\emptyset \neq T \subseteq N$ and $c>0$. We prove uniqueness of $f\left(N, c u_{T}, D\right)$ by induction on the depth $l(D)$ of the directed tree $(N, D)$. If $l(D)=0$, we have that $N=T=\left\{i_{0}\right\}$. Then EFF implies that $f_{i_{0}}\left(N, c u_{T}, D\right)=c$. Proceeding by induction, assume that for every $N^{\prime} \subseteq N$, every $T^{\prime} \subseteq N^{\prime}$, and every $\left(N^{\prime}, D^{\prime}\right), f\left(N^{\prime}, c u_{T^{\prime}}, D^{\prime}\right)$ is uniquely determined whenever $l\left(D^{\prime}\right)<l(D){ }^{22}$ For every $i \in N$, we define the set of subordinates of $i$ with rank equal to the depth of the directed tree, i.e.,

$$
H^{D}(i)=\left\{j \in \widehat{S}_{D}(i): l(j)=l(D)\right\} .
$$

We distinguish five cases with respect to $i \in N$.

Case I: $i \in N \backslash T$. By NPP, $f_{i}\left(N, c u_{T}, D\right)=0$.

Case II: $i \in T, S_{D}(i) \neq \emptyset$, and $H^{D}(i)=\emptyset$. By IMT, $f_{i}\left(N, c u_{T}, D\right)=f_{i}\left(N_{i},\left(c u_{T}\right)_{i}, D_{N_{i}}\right)$. ${ }^{23}$ Due to Lemma B.3, $\left(N_{i},\left(c u_{T}\right)_{i}\right)$ is a scaled unanimity game and, by definition of $N_{i}, l\left(D_{N_{i}}\right)<l(D)$. Then, it follows from the induction hypothesis that $f_{i}\left(N_{i},\left(c u_{T}\right)_{i}, D_{N_{i}}\right)$, and thus $f_{i}\left(N, c u_{T}, D\right)$, is uniquely determined.

Case III: $i \in T, S_{D}(i)=\emptyset$, and $l(i)<l(D)$. Following exactly the same argumentation as in Case II, it can be shown that $f_{i}\left(N, c u_{T}, D\right)$ is uniquely determined.

Case IV: $i \in T$ and $H^{D}(i) \neq \emptyset$. Note that $H^{D}(i) \neq \emptyset$ implies $S_{D}(i) \neq \emptyset$. By IMT, we have $f_{i}\left(N, c u_{T}, D\right)=f_{i}\left(N_{i},\left(c u_{T}\right)_{i}, D_{N_{i}}\right)$. However, unlike in Case II, we have $l\left(D_{N_{i}}\right)=l(D)$. Define $Q_{i}=S_{D}\left(\widehat{P}_{D}(i)\right) \backslash\left(\widehat{P}_{D}(i) \cup i\right)$ as the set of subordinates of superiors of $i$, except $i$ and all her superiors. Note that $\left\{\widehat{S}_{D}(i),\{i\}, \widehat{P}_{D}(i), Q_{i}\right\}$ is a partition of $N_{i}$. Therefore, by EFF,

$$
\begin{aligned}
& f_{i}\left(N_{i},\left(c u_{T}\right)_{i}, D_{N_{i}}\right) \\
& =c-\sum_{j \in \widehat{S}_{D}(i)} f_{j}\left(N_{i},\left(c u_{T}\right)_{i}, D_{N_{i}}\right)-\sum_{j \in \widehat{P}_{D}(i)} f_{j}\left(N_{i},\left(c u_{T}\right)_{i}, D_{N_{i}}\right)-\sum_{j \in Q_{i}} f_{j}\left(N_{i},\left(c u_{T}\right)_{i}, D_{N_{i}}\right) .
\end{aligned}
$$

For each $j \in Q_{i}$, by Lemma B.3 we have that $\left(\left(N_{i}\right)_{j},\left(\left(c u_{T}\right)_{i}\right)_{j}, D_{\left(N_{i}\right)_{j}}\right)$ is also a unanimity game and, by construction of $\left(N_{i}\right)_{j}$, we obtain that $l\left(D_{\left(N_{i}\right)_{j}}\right)<l\left(D_{N_{i}}\right)=l(D)$. The latter holds since $S_{D}(i) \neq \emptyset$ and $l^{D}(j) \leq l^{D}(i)$ for any $j \in Q_{i} \subseteq N_{i}$. Then, by applying IMT to $\left(N_{i},\left(c u_{T}\right)_{i}, D_{N_{i}}\right)$ we obtain that $f_{j}\left(N_{i},\left(c u_{T}\right)_{i}, D_{N_{i}}\right)=f_{j}\left(\left(N_{i}\right)_{j},\left(\left(c u_{T}\right)_{i}\right)_{j}, D_{\left(N_{i}\right)_{j}}\right)$ for each $j \in Q_{i}$. From the induction hypothesis, it then follows that

$$
f_{j}\left(N_{i},\left(c u_{T}\right)_{i}, D_{N_{i}}\right) \text { is uniquely determined for } j \in Q_{i} \text {. }
$$

\footnotetext{
${ }^{22}$ Note that we assume the induction hypothesis for every subset $N^{\prime}$ of $N$ and the (scaled) unanimity game on every subset $T^{\prime}$ of $N^{\prime}$.

${ }^{23}$ Throughout the proof, every time we apply IMT, we are actually using the result from Lemma B.1.
} 
We note that $\left(N_{i}\right)_{j} \cap \widehat{S}_{D}(i)=\emptyset$ for $j \in Q_{i}$. By NPP, SLB, and SUB, we have that

$$
\sum_{j \in \widehat{S}_{D}(i)} f_{j}\left(N_{i},\left(c u_{T}\right)_{i}, D_{N_{i}}\right)= \begin{cases}f_{i}\left(N_{i},\left(c u_{T}\right)_{i}, D_{N_{i}}\right) & \text { if } \widehat{S}_{D}(i) \cap T \neq \emptyset \\ 0 & \text { otherwise. }\end{cases}
$$

Let $\mathcal{X}_{i}=1$ if $\widehat{S}_{D}(i) \cap T \neq \emptyset$ and $\mathcal{X}_{i}=0$ otherwise. Next, we show uniqueness of $f_{i}\left(N_{i},\left(c u_{T}\right)_{i}, D_{N_{i}}\right)$ by a second induction on $\left|\widehat{P}_{D}(i) \cap T\right|$. First, assume that $\left|\widehat{P}_{D}(i) \cap T\right|=0$. Then, Eq. (B.21) reduces to

$$
\left(1+\mathcal{X}_{i}\right) \cdot f_{i}\left(N_{i},\left(c u_{T}\right)_{i}, D_{N_{i}}\right)=c-\sum_{j \in Q_{i}} f_{j}\left(N_{i},\left(c u_{T}\right)_{i}, D_{N_{i}}\right)
$$

Hence, $f_{i}\left(N_{i},\left(c u_{T}\right)_{i}, D_{N_{i}}\right)$ is uniquely determined.

Second, suppose that for some integer $t>0, f_{i}\left(N_{i},\left(c u_{T}\right)_{i}, D_{N_{i}}\right)$ is uniquely determined for every $i^{\prime} \in T$ with $H^{D}\left(i^{\prime}\right) \neq \emptyset$ and $\left|\widehat{P}_{D}\left(i^{\prime}\right) \cap T\right|<t$, and let $i \in T$ be such that $H^{D}(i) \neq \emptyset$ and $\left|\widehat{P}_{D}(i) \cap T\right|=t$. Take $k \in \widehat{P}_{D}(i) \cap T$ such that for every $j \in \widehat{P}_{D}(i) \cap T$ we have $l(k) \geq l(j)$, i.e., $k$ is the predecessor of $i$ who is closest to the latter in the tree among those superiors of $i$ that belong to $T$. By SLB and SUB, and the fact that $i \in T \cap \widehat{S}_{D}(k)$, we obtain

$$
\begin{aligned}
f_{k}\left(N_{i},\left(c u_{T}\right)_{i}, D_{N_{i}}\right) & =f_{i}\left(N_{i},\left(c u_{T}\right)_{i}, D_{N_{i}}\right)+\sum_{j \in \widehat{S}_{D}(i)} f_{j}\left(N_{i},\left(c u_{T}\right)_{i}, D_{N_{i}}\right) \\
& +\sum_{j \in Q_{i} \cap \widehat{S}_{D}(k)} f_{j}\left(N_{i},\left(c u_{T}\right)_{i}, D_{N_{i}}\right)+\sum_{j \in \widehat{P}_{D}(i) \cap \widehat{S}_{D}(k)} f_{j}\left(N_{i},\left(c u_{T}\right)_{i}, D_{N_{i}}\right) .
\end{aligned}
$$

Moreover, due to NPP and the definition of $k$, Eq. (B.24) reduces to

$$
\begin{aligned}
f_{i}\left(N_{i},\left(c u_{T}\right)_{i}, D_{N_{i}}\right) & =f_{k}\left(N_{i},\left(c u_{T}\right)_{i}, D_{N_{i}}\right)-\sum_{j \in \widehat{S}_{D}(i)} f_{j}\left(N_{i},\left(c u_{T}\right)_{i}, D_{N_{i}}\right) \\
& -\sum_{j \in Q_{i} \cap \widehat{S}_{D}(k)} f_{j}\left(N_{i},\left(c u_{T}\right)_{i}, D_{N_{i}}\right) .
\end{aligned}
$$

By definition of $k$, we also have $\left|\widehat{P}_{D}(k) \cap T\right|<\left|\widehat{P}_{D}(i) \cap T\right|=t$. Hence, by the second induction hypothesis, $f_{k}\left(N_{i},\left(c u_{T}\right)_{i}, D_{N_{i}}\right)$ is uniquely determined. Then, using Eq. (B.23), we can rewrite Eq. (B.25) as

$$
\left(1+\mathcal{X}_{i}\right) \cdot f_{i}\left(N_{i},\left(c u_{T}\right)_{i}, D_{N_{i}}\right)=f_{k}\left(N_{i},\left(c u_{T}\right)_{i}, D_{N_{i}}\right)-\sum_{j \in Q_{i} \cap \widehat{S}_{D}(k)} f_{j}\left(N_{i},\left(c u_{T}\right)_{i}, D_{N_{i}}\right) .
$$

Therefore, $f_{i}\left(N_{i},\left(c u_{T}\right)_{i}, D_{N_{i}}\right)$ is uniquely determined.

Case V: $i \in T, S_{D}(i)=\emptyset$, and $l(i)=l(D)$. Note that $S_{D}(i)=\emptyset$ implies $H^{D}(i)=\emptyset$. By Iмт,

$$
f_{i}\left(N, c u_{T}, D\right)=f_{i}\left(N_{i},\left(c u_{T}\right)_{i}, D_{N_{i}}\right) .
$$


Note that every $j \in N_{i} \backslash A(i)$ belongs to one of the four cases above with respect to $\left(N_{i},\left(c u_{T}\right)_{i}, D_{N_{i}}\right)$. Then, from the previous cases, the fact that $l\left(D_{N_{i}}\right)=l(D)$, and the induction hypothesis

$$
f_{j}\left(N_{i},\left(c u_{T}\right)_{i}, D_{N_{i}}\right) \text { is uniquely determined for } j \in N_{i} \backslash A(i) \text {. }
$$

Next, by EFF,

$$
\sum_{j \in A(i)} f_{j}\left(N_{i},\left(c u_{T}\right)_{i}, D_{N_{i}}\right)=c-\sum_{j \in N_{i} \backslash A(i)} f_{j}\left(N_{i},\left(c u_{T}\right)_{i}, D_{N_{i}}\right) .
$$

Further, by NPP and NSP, for each $j \in A(i)$

$$
f_{j}\left(N_{i},\left(c u_{T}\right)_{i}, D_{N_{i}}\right)= \begin{cases}f_{i}\left(N_{i},\left(c u_{T}\right)_{i}, D_{N_{i}}\right) & \text { if } j \in T \\ 0 & \text { otherwise }\end{cases}
$$

Let $\mathcal{Y}_{j}=1$ if $j \in T$ and $\mathcal{Y}_{j}=0$ otherwise. Then, from the two above equations, it follows that

$$
f_{i}\left(N_{i},\left(c u_{T}\right)_{i}, D_{N_{i}}\right) \cdot \sum_{j \in A(i)} \mathcal{Y}_{j}=c-\sum_{j \in N_{i} \backslash A(i)} f_{j}\left(N_{i},\left(c u_{T}\right)_{i}, D_{N_{i}}\right) .
$$

Uniqueness of $f_{i}\left(N, c u_{T}, D\right)$ is obtained straightforwardly by applying Eq. (B.27) to the above equation, as $\mathcal{Y}_{i}=1$.

We conclude from Cases $\mathrm{I}-\mathrm{V}$ that $f\left(N, c u_{T}, D\right)$ is uniquely determined if $c>0$. Now, consider $\left(N, c u_{T}, D\right)$ with $c<0 .^{24}$ We have already mentioned that NPP implies that $f_{i}\left(N, v_{0}, D\right)=0$ for all $i \in N$, where $\left(N, v_{0}\right)$ is the null game. Since $c u_{T}+\left(-c u_{T}\right)=v_{0}$, ADD implies that

$$
f\left(N, c u_{T}, D\right)=f\left(N, v_{0}, D\right)-f\left(N,-c u_{T}, D\right)=-f\left(N,-c u_{T}, D\right) .
$$

Since $-c>0, f\left(N,-c u_{T}, D\right)$ is uniquely determined. Thus, $f\left(N, c u_{T}, S\right)=-f\left(N,-c u_{T}, S\right)$ is also uniquely determined if $c<0$.

Finally, by ADD we have that $f(N, v, D)=\sum_{\emptyset \neq T \subseteq N} f\left(N, \Delta_{v}(T) u_{T}, D\right)$ is uniquely determined for all $(N, v) \in \mathcal{G}$.

Existence: First, solution $\varphi^{S L}$ satisfying EFF, ADD, and NPP follows from the fact that for a given $(N, v, D) \in \mathcal{G D}$, the levels structure $\left(N, B^{D}\right)$ does not depend on the game $(N, v)$, and from the properties Efficiency, Additivity, and Dummy Player Property satisfied by the Shapley Levels Value (see Winter, 1989).

\footnotetext{
${ }^{24}$ Note that we cannot apply the superior upper and lower bound properties, since $c u_{T}$ is not monotone if $c<0$.
} 
To show that $\varphi^{S L}$ also satisfies IMT, let $(N, v, D) \in \mathcal{G D}$ and $i \in N$. Let also $U_{r, s}^{i} \in B_{r, s}^{D}$ be such that $i \in U_{r, s}^{i}$, with $(r, s) \in\{1, \ldots, l(D)\} \times\{1,2\}$. Then,

$$
U_{r, l}^{i}= \begin{cases}U \supseteq \widehat{S}_{D}(i) \cup i & \text { if } r<l(i) \text { or }(r, s)=(l(i), 1), \\ \widehat{S}_{D}(i) \cup i & \text { if }(r, l)=(l(i), 2), \\ \{i\} & \text { if } r>l(i) .\end{cases}
$$

Moreover, $\widehat{S}_{D}(i) \in B_{l(i)+1,1}^{D}$. That is, $i$ and her subordinates belong to the same union in all levels of $\left(N, B^{D}\right)$ prior to level $(l(i), 2)$, at which point the union that contains $i$ is exactly $\widehat{S}_{D}(i) \cup i$, with $\widehat{S}_{D}(i)$ and $\{i\}$ being unions of the next level. From then on, $\{i\}$ is always a union herself.

In Álvarez-Mozos et al. (2013), a Multiplication Property satisfied by the Shapley Levels Value is identified. Here, we only provide an informal description of the property to avoid the introduction of further notation. ${ }^{25}$ The property requires the share of $v(N)$ - prescribed by the Shapley Levels Valuethat a player obtains in a game with levels structure be obtained by multiplying each of the shares received according to the Shapley Value by each of the unions $U_{r, l}^{i}$ in certain internal games defined for each player and each level of the levels structure. The internal game that corresponds to level $(r, l)$ only uses the information contained in the unions of the coarser levels to which the unions of the game belong, i.e. $U_{k, h}^{i}$, with $(k, h) \leq_{l e x}(r, l)$, and disregards any other information. For example, with only one level of cooperation there is an internal game played by all unions and then, there are as many internal games as unions in the partition, and the player set of each of these games is the corresponding union. For every union, the internal game then describes the prospects of a coalition that defects from the union to form a union itself. From the fact that the Shapley Levels Value satisfies the Multiplication Property, it follows from Eq. (B.28) that, due to the way it is constructed, $\varphi^{S L}$ satisfies IMT.

To show that $\varphi^{S L}$ satisfies NSP, let $(N, v, D) \in \mathcal{G D}$ and $i \in N$ with $S_{D}(i)=\emptyset$. Then, it can be easily verified that every $j \in A(i)$ with $S_{D}(j)=\emptyset$ has a completely symmetric position in the structure $\left(N, B^{D}\right)$ w.r.t. player $i$, meaning that for every level, both $i$ and $j$ either belong to the same union or each of them forms a union as a singleton. From this observation, it follows that in exactly half of the permutations in $\Omega\left(B^{D}\right), i$ comes before $j$ and vice versa. Then, whenever $j$ is a necessary player and $(N, v) \in \mathcal{G}_{M}$, we obtain from Eq. (2.1) and the definition of $\varphi^{S L}$ that $f_{i}(N, v, D) \leq f_{j}(N, v, D)$.

Finally, to show that $\varphi^{S L}$ satisfies SLB and SUB, we use the Level game property fulfilled by the Shapley Levels Value (Álvarez-Mozos and Tejada, 2011). Let $(N, v, \underline{B})$ be a game with levels structure of cooperation and let $T \in B_{r} \in \underline{B}$. This property states that the joint payoff to the members of

\footnotetext{
${ }^{25}$ For a formal description, we refer to Álvarez-Mozos et al. (2013).
} 
$T$ according to the Shapley Levels Value is precisely the payoff to the union $T$ in a game with levels structure where the players are the unions at level $r$ and the structure is obtained from $\underline{B}$ by truncating its levels at level $r$. That is, $\sum_{i \in T} S h_{i}^{L}(N, v, \underline{B})=S h_{T}^{L}\left(B_{r}, v^{r},\left(B_{1}, \ldots, B_{r}\right)\right)$, where for every $Q \subseteq B_{r}$, $v^{r}(Q)=v\left(\cup_{R \in Q} R\right)$.

Let $(N, v, D) \in \mathcal{G D}$ and $i \in N$. Then, note that Eq. (B.28) implies that among the admissible permutations of $\Omega\left(B^{D}\right), i$ comes before her subordinates in half of them and in the other half, all her subordinates come before player $i$. By the Level game property described above, it is enough to study the payoffs to the unions up to level $(l(i)+1,1)$. Following a reasoning similar to the one used for NSP, we can conclude that if $i$ is a necessary player in a monotone game, then she earns as much as all her subordinates together and vice versa. Thus, $\varphi^{S L}$ satisfies both SLB and SUB. 


\section{Appendix C Proofs of Section 5}

\section{Proof of Theorem II}

We first show uniqueness and then existence.

Uniqueness: The proof follows the same steps as in the proof of Theorem I, except for Case IV, where we use TLB and TUB instead of SLB and SUB. Therefore, we only show how to adapt Case IV. Accordingly, let $i \in T$ be such that $H^{D}(i) \neq \emptyset$. Proceeding as in the proof of Theorem I, Eqs. (B.21) and (B.22) follow. Then, by applying NPP, TLB, and TUB, instead of Eq. (B.23) we obtain for all $j \in S_{D}(i)$

$$
\sum_{h \in \widehat{S}_{D}(j) \cup j} f_{h}\left(N_{i},\left(c u_{T}\right)_{i}, D_{N_{i}}\right)= \begin{cases}f_{i}\left(N_{i},\left(c u_{T}\right)_{i}, D_{N_{i}}\right) & \text { if }\left(\widehat{S}_{D}(j) \cup j\right) \cap T \neq \emptyset, \\ 0 & \text { otherwise. }\end{cases}
$$

Now, for each $j \in S_{D}(i)$, let $\mathcal{Z}_{j}=1$ if $\left(\widehat{S}_{D}(j) \cup j\right) \cap T \neq \emptyset$ and $\mathcal{Z}_{j}=0$ otherwise. Next, we conduct a second induction on $\left|\widehat{P}_{D}(i) \cap T\right|$ as in the proof of Theorem I. When $\left|\widehat{P}_{D}(i) \cap T\right|=0$, Eq. (B.21) now reduces to

$$
\left(1+\sum_{j \in S_{D}(i)} \mathcal{Z}_{j}\right) \cdot f_{i}\left(N_{i},\left(c u_{T}\right)_{i}, D_{N_{i}}\right)=c-\sum_{j \in Q_{i}} f_{j}\left(N_{i},\left(c u_{T}\right)_{i}, D_{N_{i}}\right),
$$

so $f_{i}\left(N_{i},\left(c u_{T}\right)_{i}, D_{N_{i}}\right)$ is uniquely determined.

Now, suppose that for some integer $t>0, f_{i}\left(N_{i},\left(c u_{T}\right)_{i}, D_{N_{i}}\right)$ is uniquely determined for every $i^{\prime} \in T$ with $H^{D}\left(i^{\prime}\right) \neq \emptyset$ and $\left|\widehat{P}_{D}\left(i^{\prime}\right) \cap T\right|<t$, and assume that $\left|\widehat{P}_{D}(i) \cap T\right|=t$. Take $k \in \widehat{P}_{D}(i) \cap T$ such that for every $j \in \widehat{P}_{D}(i) \cap T$, we have $l(k) \geq l(j)$, i.e., $k$ is the superior of $i$ who is closest to the latter in the tree among those superiors of $i$ that belong to $T$. Let also $p \in S_{D}(k) \cap\left(\widehat{P}_{D}(i) \cup i\right)$, and note that $p$ is uniquely defined. By TLB and TUB, and the fact that $i \in T$, we obtain

$$
\begin{aligned}
f_{k}\left(N_{i},\left(c u_{T}\right)_{i}, D_{N_{i}}\right) & =f_{i}\left(N_{i},\left(c u_{T}\right)_{i}, D_{N_{i}}\right)+\sum_{j \in \widehat{S}_{D}(i)} f_{j}\left(N_{i},\left(c u_{T}\right)_{i}, D_{N_{i}}\right) \\
& +\sum_{j \in Q_{i} \cap \widehat{S}_{D}(p)} f_{j}\left(N_{i},\left(c u_{T}\right)_{i}, D_{N_{i}}\right)+\sum_{j \in \widehat{P}_{D}(i) \cap\left(\widehat{S}_{D}(p) \cup p\right)} f_{j}\left(N_{i},\left(c u_{T}\right)_{i}, D_{N_{i}}\right) .
\end{aligned}
$$

Moreover, due to NPP, Eq. (C.30) reduces to

$$
\begin{aligned}
& f_{i}\left(N_{i},\left(c u_{T}\right)_{i}, D_{N_{i}}\right) \\
= & f_{k}\left(N_{i},\left(c u_{T}\right)_{i}, D_{N_{i}}\right)-\sum_{j \in \widehat{S}_{D}(i)} f_{j}\left(N_{i},\left(c u_{T}\right)_{i}, D_{N_{i}}\right)-\sum_{j \in Q_{i} \cap \widehat{S}_{D}(k)} f_{j}\left(N_{i},\left(c u_{T}\right)_{i}, D_{N_{i}}\right) \\
= & f_{k}\left(N_{i},\left(c u_{T}\right)_{i}, D_{N_{i}}\right)-\sum_{j \in S_{D}(i)} \sum_{h \in\left(\widehat{S}_{D}(j) \cup j\right)} f_{h}\left(N_{i},\left(c u_{T}\right)_{i}, D_{N_{i}}\right)-\sum_{j \in Q_{i} \cap \widehat{S}_{D}(k)} f_{j}\left(N_{i},\left(c u_{T}\right)_{i}, D_{N_{i}}\right) .
\end{aligned}
$$


By definition of $k$, we have $\left|\widehat{P}_{D}(k) \cap T\right|<\left|\widehat{P}_{D}(i) \cap T\right|=t$. Hence, by the second induction hypothesis, $f_{k}\left(N_{i},\left(c u_{T}\right)_{i}, D_{N_{i}}\right)$ is uniquely determined. By using Eq. (C.29), we can rewrite Eq. (C.31) as

$$
\left(1+\sum_{j \in S_{D}(i)} \mathcal{Z}_{j}\right) \cdot f_{i}\left(N_{i},\left(c u_{T}\right)_{i}, D_{N_{i}}\right)=f_{k}\left(N_{i},\left(c u_{T}\right)_{i}, D_{N_{i}}\right)-\sum_{j \in Q_{i} \cap \widehat{S}_{D}(k)} f_{j}\left(N_{i},\left(c u_{T}\right)_{i}, D_{N_{i}}\right) .
$$

Therefore, $f_{i}\left(N_{i},\left(c u_{T}\right)_{i}, D_{N_{i}}\right)$ is uniquely determined. This completes Case IV. The rest of the uniqueness part of the proof is done in the same way as the proof of Theorem I.

Existence: Solution $\bar{\varphi}^{S L}$ satisfying EFF, ADD, and NPP can be shown as in the proof of Theorem I. To prove that $\bar{\varphi}^{S L}$ also satisfies IMT, let $(N, v, D) \in \mathcal{G D}$ and $i \in N$. Note that when $l(i)<l(D)$, it holds that

$$
\begin{aligned}
& \widehat{S}_{D}(i) \cup i \in B_{l(i), 2}^{D} \text { and for every } j \in S_{D}(i), \\
& \widehat{S}_{D}(j) \cup j \in B_{l(i)+1,2}^{D} .
\end{aligned}
$$

That is, $i$ and her subordinates belong to the same union in all levels of $\left(N, \bar{B}^{D}\right)$ prior to level $l(i)$, at which point $i$ and her successors' teams form separate unions. From then on, $i$ is always a singleton. From the fact that the Shapley Levels Value satisfies the multiplication property (Álvarez-Mozos et al., 2013), it can be verified due to Eq. (C.32) that because of the way it is constructed, $\bar{\varphi}^{S L}$ satisfies IMT. To show that $\bar{\varphi}^{S L}$ satisfies NSP, we can repeat the argument used in the proof of Theorem I, since two siblings with no successors also have a symmetric position in the levels structure $\left(N, \bar{B}^{D}\right)$. Finally, to show that $\bar{\varphi}^{S L}$ satisfies TLB and TUB we can replicate the argument used in Theorem I to show the subordinate bounds using Eq. (C.32) instead of Eq. (B.28).

\section{Proof of Theorem III}

We first show uniqueness and then existence.

Uniqueness: Suppose that $f$ satisfies the seven axioms. For every $\left(N, v_{0}, D\right)$, with $\left(N, v_{0}\right)$ being the null game, NPP implies that $f_{i}\left(N, v_{0}, D\right)=0$ for all $i \in N$. Next, let $\left(N, c u_{T}, D\right) \in \mathcal{G D}$, where $\emptyset \neq T \subseteq N$ and $c>0$. For every $i \in N \backslash T$, by NPP, $f_{i}\left(N, c u_{T}, D\right)=0$.

Let $i \in T$ henceforth. If $|T|=1$, NPP and EFF imply that $f_{i}(N, v, D)=c$. Hence, assume that $|T| \geq 2$. We show uniqueness of $f_{i}\left(N, c u_{T}, D\right)$ by induction on her rank, $l(i)$. If $l(i)=0$, by using EFF, SSLB, and SSUB, we easily obtain that $f_{i}\left(N, c u_{T}, D\right)=\frac{c}{2}$. Then, assume that for every $j \in T$ with $l(j)<l, f_{j}\left(N, c u_{T}, D\right)$ is uniquely determined, and consider that $l(i)=l$ for some integer $l \in\{1, \ldots, l(D)\}$. For each $j \in N$, let $\mathcal{X}_{j}=1$ if $\widehat{S}_{D}(j) \cap T \neq \emptyset$ and $\mathcal{X}_{j}=0$ otherwise. Also, for each $j \in N$, let $\mathcal{Y}_{j}=1$ if $j \in T$ and $\mathcal{Y}_{j}=0$ otherwise. 
By NPP, SSLB, and SSUB, we have that for each $j \in A(i)$

$$
\sum_{h \in \widehat{S}_{D}(j)} f_{h}(N, v, D)=\mathcal{X}_{j} \cdot f_{i}(N, v, D) .
$$

Similarly, by NPP and SNSP, we have that

$$
\sum_{j \in A(i)} f_{j}(N, v, D)=\sum_{j \in A(i)} \mathcal{Y}_{j} \cdot f_{i}(N, v, D)
$$

Next, we distinguish two cases, depending on whether $l=1$ or $l>1$.

Case I: $l=1$. Note that we have $i_{0}=p_{D}(i)$. By EFF, we obtain

$$
c=f_{i_{0}}(N, v, D)+\sum_{j \in A(i)}\left(f_{j}(N, v, D)+\sum_{h \in \widehat{S}_{D}(j)} f_{h}(N, v, D)\right) .
$$

By using Eqs. (C.33) and (C.34), the above equation reduces to

$$
c=f_{i_{0}}(N, v, D)+\sum_{j \in A(i)}\left(\mathcal{Y}_{j}+\mathcal{X}_{j}\right) \cdot f_{i}(N, v, D)
$$

By the induction hypothesis, $f_{i_{0}}(N, v, D)$ is determined. Moreover, the coefficient of $f_{i}(N, v, D)$ in the above equation is strictly positive. Thus, $f_{i}(N, v, D)$ is determined.

Case II: $l>1$. For every $r \in\{0, \ldots, l\}$, let $i_{r} \in\left(\widehat{P}_{D}(i) \cup i\right)$ be such that $l\left(i_{r}\right)=r$. Note that, in particular, we have $i_{l}=i$. Also note that $\mathcal{Y}_{i_{l}}=1$ and $\mathcal{X}_{i_{r}}=1$ for all $r \in\{0, \ldots, l-1\}$.

First, by NPP and NOP, for each $r \in\{1, \ldots, l-1\}$,

$$
\sum_{j \in A\left(i_{r}\right)} \sum_{h \in \widehat{S}_{D}(j)} f_{h}(N, v, D)=\sum_{j \in A\left(i_{r}\right)} \mathcal{X}_{j} \cdot \sum_{j \in \widehat{S}_{D}\left(i_{r}\right)} f_{j}(N, v, D)
$$

Second, we claim that for $r \in\{0, \ldots, l\},{ }^{26}$

$$
c=\sum_{s=0}^{r}\left[\prod_{p=0}^{s-1} \sum_{j \in A\left(i_{p}\right)} \mathcal{X}_{j} \cdot \sum_{j \in A\left(i_{s}\right)} f_{j}(N, v, D)\right]+\prod_{p=0}^{r} \sum_{j \in A\left(i_{p}\right)} \mathcal{X}_{j} \cdot \sum_{j \in \widehat{S}_{D}\left(i_{r}\right)} f_{j}(N, v, D) .
$$

We prove Eq. (C.36) by induction on $r$. The case $r=0$ is straightforward, since due to EFF, $c=$ $f_{i_{0}}(N, v, D)+\sum_{j \in \widehat{S}_{D}\left(i_{0}\right)} f_{j}(N, v, D)$. Hence, assume that Eq. (C.36) holds if we substitute $r$ by $r-1$.

\footnotetext{
${ }^{26}$ The multiplication over an empty set is 1 .
} 
To facilitate the presentation of the calculations, we denote $f_{j}=f_{j}(N, v, D)$ for all $j \in N$. Then,

$$
\begin{aligned}
& \sum_{s=0}^{r}\left[\prod_{p=0}^{s-1} \sum_{j \in A\left(i_{p}\right)} \mathcal{X}_{j} \cdot \sum_{j \in A\left(i_{s}\right)} f_{j}\right]+\prod_{p=0}^{r} \sum_{j \in A\left(i_{p}\right)} \mathcal{X}_{j} \cdot \sum_{j \in \widehat{S}_{D}\left(i_{r}\right)} f_{j} \\
= & \sum_{s=0}^{r-1}\left[\prod_{p=0}^{s-1} \sum_{j \in A\left(i_{p}\right)} \mathcal{X}_{j} \cdot \sum_{j \in A\left(i_{s}\right)} f_{j}\right]+\prod_{p=0}^{r-1} \sum_{j \in A\left(i_{p}\right)} \mathcal{X}_{j} \cdot \sum_{j \in \widehat{S}_{D}\left(i_{r-1}\right)} f_{j} \\
+ & \prod_{p=0}^{r-1} \sum_{j \in A\left(i_{p}\right)} \mathcal{X}_{j} \cdot \sum_{j \in A\left(i_{r}\right)} f_{j}+\prod_{p=0}^{r} \sum_{j \in A\left(i_{p}\right)} \mathcal{X}_{j} \cdot \sum_{j \in \widehat{S}_{D}\left(i_{r}\right)} f_{j}-\prod_{p=0}^{r-1} \sum_{j \in A\left(i_{p}\right)} \mathcal{X}_{j} \cdot \sum_{j \in \widehat{S}_{D}\left(i_{r-1}\right)} f_{j} \\
= & c+\prod_{p=0}^{r-1} \sum_{j \in A\left(i_{p}\right)} \mathcal{X}_{j} \cdot\left(\sum_{j \in A\left(i_{r}\right)} f_{j}+\sum_{j \in A\left(i_{r}\right)} \mathcal{X}_{j} \cdot \sum_{j \in \widehat{S}_{D}\left(i_{r}\right)} f_{j}-\sum_{j \in \widehat{S}_{D}\left(i_{r-1}\right)} f_{j}\right)=c,
\end{aligned}
$$

where the second equality holds from the second induction hypothesis and the last equality is explained as follows. Indeed, note that for every $r \in\{1, \ldots, l-1\}$

$$
\begin{aligned}
& \sum_{j \in A\left(i_{r}\right)} f_{j}+\sum_{j \in A\left(i_{r}\right)} \mathcal{X}_{j} \cdot \sum_{j \in \widehat{S}_{D}\left(i_{r}\right)} f_{j}-\sum_{j \in \widehat{S}_{D}\left(i_{r-1}\right)} f_{j} \\
= & \sum_{j \in A\left(i_{r}\right)} f_{j}+\sum_{j \in A\left(i_{r}\right)} \mathcal{X}_{j} \cdot \sum_{j \in \widehat{S}_{D}\left(i_{r}\right)} f_{j}-\sum_{j \in A\left(i_{r}\right)} f_{j}-\sum_{j \in A\left(i_{r}\right)} \sum_{h \in \widehat{S}_{D}(j)} f_{h}=0,
\end{aligned}
$$

where the last equality holds by Eq. (C.35).

Third, by using Eq. (C.36) when $r=l$, Eq. (C.36) reduces to

$$
c=\sum_{s=0}^{l-1}\left[\prod_{p=0}^{s-1} \sum_{j \in A\left(i_{p}\right)} \mathcal{X}_{j} \cdot \sum_{j \in A\left(i_{s}\right)} f_{j}\right]+\prod_{p=0}^{l-1} \sum_{j \in A\left(i_{p}\right)} \mathcal{X}_{j} \cdot \sum_{j \in A(i)} f_{j}+\prod_{p=0}^{l} \sum_{j \in A\left(i_{p}\right)} \mathcal{X}_{j} \cdot \sum_{j \in \widehat{S}_{D}(i)} f_{j} .
$$

Fourth, applying Eqs. (C.33) and (C.34) to Eq. (C.37) yields

$$
c=\sum_{s=0}^{l-1}\left[\prod_{p=0}^{s-1} \sum_{j \in A\left(i_{p}\right)} \mathcal{X}_{j} \cdot \sum_{j \in A\left(i_{s}\right)} f_{j}\right]+\left[\prod_{p=0}^{l-1} \sum_{j \in A\left(i_{p}\right)} \mathcal{X}_{j} \cdot \sum_{j \in A(i)} \mathcal{Y}_{j}+\prod_{p=0}^{l} \sum_{j \in A\left(i_{p}\right)} \mathcal{X}_{j} \cdot \mathcal{X}_{i}\right] \cdot f_{i}(N, v, D) .
$$

By the first induction hypothesis, the first term in the right-hand side of Eq. (C.38) is determined. Moreover, the coefficient of $f_{i}(N, v, D)$ in Eq. (C.38) is strictly positive. Therefore, $f_{i}(N, v, D)$ is unique.

Thus, we have shown that $f\left(N, c u_{T}, D\right)$ is uniquely determined. Following the lines of the proof of Theorem I, it can also be verified that $f\left(N, c u_{T}, D\right)$ is uniquely determined if $c<0$, hence implying by ADD that $f(N, v, D)=\sum_{\emptyset \neq T \subseteq N} f\left(N, \Delta_{v}(T) u_{T}, D\right)$ is uniquely determined for all $(N, v) \in \mathcal{G}$.

Existence: First, solution $\widetilde{\varphi}^{S L}$ satisfying EFF, ADD, and NPP can be shown as in the proof of Theorem I. To prove that $\widetilde{\varphi}^{S L}$ also satisfies SNSP, SSLB, SSUB, and NOP, let $(N, v, D) \in \mathcal{G D}, i \in N$, and 
$j \in A(i)$. Then for every $l<l(i)$,

$$
\begin{aligned}
& \widehat{S}_{D}(i) \cup \widehat{S}_{D}(j) \cup\{i, j\} \subseteq T \in B_{l, 1}^{D} \text { and } \\
& \widehat{S}_{D}(i), \widehat{S}_{D}(j),\{i\},\{j\} \in B_{l(i), 1}^{D} .
\end{aligned}
$$

That is, $i$ and her siblings (as well as all their subordinates) belong to the same union in all levels of $\left(N, \widetilde{B}^{D}\right)$ prior to level $l(i)$, at which point $i$ and her siblings are all singletons, while the subordinates of each sibling of $i$ (including $i$ herself) constitute a union of that level.

To show that $\widetilde{\varphi}^{S L}$ satisfies SNSP, we can repeat the argument used in the proof of Theorem I since, by Eq. (C.39), two siblings have a symmetric position in the structure $\left(N, \widetilde{B}^{D}\right)$, even if they have successors. A similar reasoning shows that $\widetilde{\varphi}^{S L}$ satisfies SSLB, SSUB, and NOP. Indeed, from Eq. (C.39), it is easy to verify that for every $i \in N$ and $j \in A(i)$, coalitions $\{i\}$ and $\widehat{S}_{D}(j)$ have a symmetric position in the structure $\left(N, \widetilde{B}^{D}\right)$ because they are in the same union up to certain level at which both coalitions became a union of the partition. Consequently, there are as many admissible permutations in $\Omega\left(\widetilde{B}^{D}\right)$ in which $i$ comes before coalition $\widehat{S}_{D}(j)$ than admissible permutations in which $i$ comes after coalition $\widehat{S}_{D}(j)$. From this observation, it follows that $\widetilde{\varphi}^{S L}$ satisfies SSLB and SSUB. Finally, to show that $\widetilde{\varphi}^{S L}$ satisfies NOP, we note that the reasoning above also applies to the coalitions $\widehat{S}_{D}(i)$ and $\widehat{S}_{D}(j)$, given that they also have a symmetric position in the structure $\left(N, \widetilde{B}^{D}\right)$. 


\section{Appendix D Logical independence of the axioms}

\section{Independence of the axioms of Theorem I}

1. The solution $f_{i}(N, v, D)=0$ for all $(N, v, D) \in \mathcal{G D}$ and $i \in N$ satisfies ADD, NPP, IMT, NSP, SLB, and SUB. It does not satisfy EFF.

2. The solution $f(N, v, D)=v(N) \varphi^{S L}\left(N, u_{E(N, v)}, D\right)$ for all $(N, v, D) \in \mathcal{G D}$, with $E(N, v)$ being the set of all non-null players in $(N, v)$, satisfies EFF, NPP, IMT, NSP, SLB, and SUB. It does not satisfy ADD.

3. Let $\omega \in \mathbb{R}_{++}^{\Omega}$ be an exogenous vector. For a given $(N, v, D) \in \mathcal{G D}$, let

$$
R=\left\{i \in N: i \text { is not a null player and } S_{D}(A(i))=\emptyset\right\}
$$

Then, consider the solution $f$ defined for each $(N, v, D) \in \mathcal{G D}$ and each $i \in N$ as follows. First, if $v=u_{T}$ for some $T \subseteq N$,

$$
f_{i}(N, v, D)= \begin{cases}\frac{\omega_{i}}{\sum_{j \in A(i) \cap R} \omega_{j}} \sum_{j \in A(i)} \varphi_{i}^{S L}(N, v, D) & \text { if } N=\Omega \text { and } i \in R \\ \varphi_{i}^{S L}(N, v, D) & \text { otherwise }\end{cases}
$$

Second, for an arbitrary $(N, v) \in \mathcal{G}, f(N, v, D)=\sum_{\emptyset \neq T \subseteq N} f\left(N, \Delta_{v}(T) u_{T}, D\right)$. Then $f$ satisfies ADD, EFF, NPP, IMT, SLB, and SUB. Moreover, $f$ does not satisfy NSP.

4. The solution $f(N, v, D)=v(N) \varphi^{S L}\left(N, u_{N}, D\right)$ for all $(N, v, D) \in \mathcal{G D}$ satisfies ADD, EFF, IMT, NSP, SLB, and SUB. It does not satisfy NPP.

5. The solution $\widetilde{\varphi}^{S L}$ introduced in Definition 5.2 satisfies ADD, EFF, NPP, NSP, SLB, and SUB. It does not satisfy IMT.

6. Recall that $i_{0}$ denotes the root of the tree. The solution given by $f_{i_{0}}(N, v, D)=v(N)-v\left(N \backslash\left\{i_{0}\right\}\right)$, and $f_{i}(N, v, D)=\varphi^{S L}\left(N,\left.v\right|_{N \backslash\left\{i_{0}\right\}}, D\right)$ if $i \in N \backslash\left\{i_{0}\right\}$, satisfies EFF, ADD, NPP, IMT, NSP, and SLB. It does not satisfy sub.

7. The solution $\bar{\varphi}^{S L}$ introduced in Definition 5.1 satisfies EFF, ADD, NPP, IMT, NSP, and SUB. It does not satisfy SLB. 


\section{Independence of the axioms of Theorem II}

1. The solution $f_{i}(N, v, D)=0$ for all $(N, v, D) \in \mathcal{G D}$ and $i \in N$ satisfies ADD, NPP, IMT, NSP, TLB, and TUB. It does not satisfy EFF.

2. The solution $f(N, v, D)=v(N) \bar{\varphi}^{S L}\left(N, u_{E(N, v)}, D\right)$ for all $(N, v, D) \in \mathcal{G D}$, with $E(N, v)$ being the set of all non-null players in $(N, v)$, satisfies EFF, NPP, IMT, NSP, TLB, and TUB. It does not satisfy ADD.

3. The solution $f(N, v, D)=v(N) \bar{\varphi}^{S L}\left(N, u_{N}, D\right)$ for all $(N, v, D) \in \mathcal{G D}$ satisfies ADD, EFF, IMT, NSP, TLB, and TUB. It does not satisfy NPP.

4. Let $\omega \in \mathbb{R}_{++}^{\Omega}$ be an exogenous vector. For a given $(N, v, D) \in \mathcal{G D}$, let

$$
R=\left\{i \in N: i \text { is not a null player and } S_{D}(A(i))=\emptyset\right\}
$$

Then, consider the solution $f$ defined for each $(N, v, D) \in \mathcal{G D}$ and each $i \in N$ as follows. First, if $v=u_{T}$ for some $T \subseteq N$,

$$
f_{i}(N, v, D)= \begin{cases}\frac{\omega_{i}}{\sum_{j \in A(i) \cap R} \omega_{j}} \sum_{j \in A(i)} \bar{\varphi}_{i}^{S L}(N, v, D) & \text { if } N=\Omega \text { and } i \in R \\ \bar{\varphi}_{i}^{S L}(N, v, D) & \text { otherwise. }\end{cases}
$$

Second, for an arbitrary $(N, v) \in \mathcal{G}, f(N, v, D)=\sum_{\emptyset \neq T \subseteq N} f\left(N, \Delta_{v}(T) u_{T}, D\right)$. Then $f$ satisfies ADD, EFF, NPP, IMT, TLB, and TUB. Moreover, $f$ does not satisfy NSP.

5. Let $N^{*}=\{1,2,3,4,5\}$ and $D^{*}=\{(1,2),(1,3),(2,4),(3,5)\}$. Let also $\alpha \in[0,1] \backslash\{0.5\}$. Then, consider the solution $f$ defined for each $\left(N, u_{T}, D\right) \in \mathcal{G D}$, with $T \subseteq N$, and $i \in N$ as follows:

$$
f_{i}\left(N, u_{\{3,4\}}, D\right)= \begin{cases}\alpha & \text { if }(N, v, D)=\left(N^{*}, u_{\{3,4\}}, D^{*}\right) \text { and } i=3, \\ 1-\alpha & \text { if }(N, v, D)=\left(N^{*}, u_{\{3,4\}}, D^{*}\right) \text { and } i=4, \\ \bar{\varphi}_{i}^{S L}\left(N, u_{T}, D\right) & \text { otherwise. }\end{cases}
$$

The solution $f$ on $\mathcal{G D}$ is then simply obtained as the additive extension on the whole class of games with hierarchical structure, and it satisfies ADD, EFF, NPP, NSP, TLB, and TUB. Moreover, $f$ does not satisfy IMT.

6. The solution $f(N, v, D)=S h(N, v)$ satisfies ADD, EFF, NPP, IMT, NSP, and TUB. It does not satisfy TLB. 
7. The solution $\varphi^{S L}$ satisfies ADD, EFF, NPP, IMT, NSP, and TLB. It does not satisfy TUB.

\section{Independence of the axioms of Theorem III}

1. The solution $f_{i}(N, v, D)=0$ for all $(N, v, D) \in \mathcal{G D}$ and $i \in N$ satisfies ADD, NPP, SNSP, SSLB, SSUB, and NOP. It does not satisfy EFF.

2. The solution $f(N, v, D)=v(N) \widetilde{\varphi}^{S L}\left(N, u_{E(N, v)}, D\right)$ for all $(N, v, D) \in \mathcal{G D}$, with $E(N, v)$ being the set of all non-null players in $(N, v)$, satisfies EFF, NPP, SNSP, SSLB, SSUB, and NOP. It does not satisfy ADD.

3. The solution $f(N, v, D)=v(N) \widetilde{\varphi}^{S L}\left(N, u_{N}, D\right)$ for all $(N, v, D) \in \mathcal{G D}$ satisfies EFF, ADD, SNSP, SSLB, SSUB, and NOP. It does not satisfy NPP.

4. Let $N^{*}=\{1,2\}$ and $D^{*}=\{(1,2)\}$, and consider the solution $f$ defined for each $\left(N, u_{T}, D\right) \in \mathcal{G D}$, with $T \subseteq N$, and $i \in N$ as follows:

$$
f_{i}\left(N, u_{T}, D\right)= \begin{cases}\frac{i}{3} & \text { if }(N, v, D)=\left(N^{*}, u_{\{1,2\}}, D^{*}\right) \text { and } i \in\{1,2\}, \\ \widetilde{\varphi}_{i}^{S L}\left(N, u_{T}, D\right) & \text { otherwise. }\end{cases}
$$

The solution $f$ on $\mathcal{G D}$ is then simply obtained as the additive extension on the entire class of games with hierarchical structure and it satisfies EFF, ADD, NPP, SNSP, SSUB, and NOP. It does not satisfy SSLB.

5. Let $N^{*}=\{1,2\}$ and $D^{*}=\{(1,2)\}$, and consider the solution $f$ defined for each $\left(N, u_{T}, D\right) \in \mathcal{G D}$, with $T \subseteq N$, and $i \in N$ as follows:

$$
f_{i}\left(N, u_{t}, D\right)= \begin{cases}\frac{3-i}{3} & \text { if }(N, v, D)=\left(N^{*}, u_{\{1,2\}}, D^{*}\right) \text { and } i \in\{1,2\} \\ \widetilde{\varphi}_{i}^{S L}\left(N, u_{T}, D\right) & \text { otherwise. }\end{cases}
$$

The solution $f$ on $\mathcal{G D}$ is then simply obtained as the additive extension on the whole class of games with hierarchical structure and it satisfies EFF, ADD, NPP, SNSP, SSLB, and NOP. It does not satisfy SSUB.

6. Let $N^{*}=\{1,2,3\}$ and $D^{*}=\{(1,2),(1,3)\}$, and consider the solution $f$ defined for each $\left(N, u_{T}, D\right) \in \mathcal{G D}$, with $T \subseteq N$, and $i \in N$ as follows:

$$
f_{i}(N, v, D)= \begin{cases}\frac{i}{5} & \text { if }(N, v, D)=\left(N^{*}, u_{\{2,3\}}, D^{*}\right) \text { and } i \in\{2,3\} \\ \widetilde{\varphi}_{i}^{S L}\left(N, u_{T}, D\right) & \text { otherwise. }\end{cases}
$$


Then, solution $f$ on $\mathcal{G D}$ is then simply obtained as the additive extension on the whole class of games with hierarchical structure and it satisfies EFF, ADD, NPP, SSUB, SSLB, and NOP. It does not satisfy SNSP.

7. Let $N^{*}=\{1,2,3,4,5\}$ and $D^{*}=\{(1,2),(1,3),(2,4),(3,5)\}$, and consider the solution $f$ defined for each $\left(N, u_{T}, D\right) \in \mathcal{G D}$, with $T \subseteq N$, and $i \in N$ as follows:

$$
f_{i}(N, v, D)= \begin{cases}\frac{i}{9} & \text { if }(N, v, D)=\left(N^{*}, u_{\{4,5\}}, D^{*}\right) \text { and } i \in\{4,5\} \\ \widetilde{\varphi}_{i}^{S L}\left(N, u_{T}, D\right) & \text { otherwise. }\end{cases}
$$

Then, solution $f$ on $\mathcal{G D}$ is then simply obtained as the additive extension on the whole class of games with hierarchical structure and it satisfies EFF, ADD, NPP, SNSP, SSUB, and SSLB. It does not satisfy NOP. 